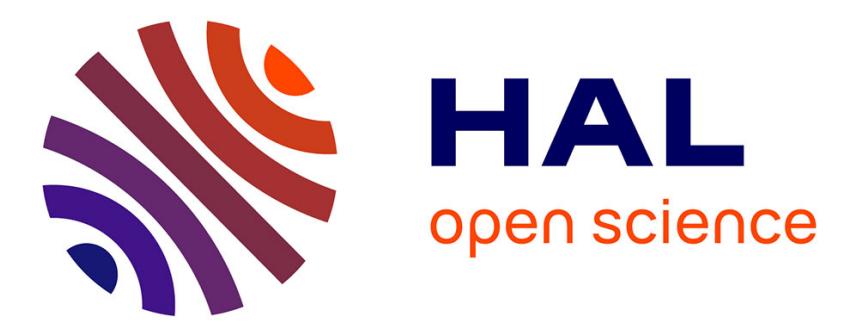

\title{
Motion of a solid particle in a shear flow along a porous slab
}

Sondes Khabthani, Antoine Sellier, Lassaad Elasmi, Francois Feuillebois

\section{To cite this version:}

Sondes Khabthani, Antoine Sellier, Lassaad Elasmi, Francois Feuillebois. Motion of a solid particle in a shear flow along a porous slab. Journal of Fluid Mechanics, 2012, 713, pp.271-306. 10.1017/jfm.2012.457 . hal-00996515

\section{HAL Id: hal-00996515 \\ https://hal-polytechnique.archives-ouvertes.fr/hal-00996515}

Submitted on 5 Jun 2014

HAL is a multi-disciplinary open access archive for the deposit and dissemination of scientific research documents, whether they are published or not. The documents may come from teaching and research institutions in France or abroad, or from public or private research centers.
L'archive ouverte pluridisciplinaire HAL, est destinée au dépôt et à la diffusion de documents scientifiques de niveau recherche, publiés ou non, émanant des établissements d'enseignement et de recherche français ou étrangers, des laboratoires publics ou privés. 


\title{
Motion of a solid particle in a shear flow along a porous slab
}

\author{
Sondes Khabthani ${ }^{1}$, Antoine Sellier ${ }^{2}$, Lassaad Elasmi ${ }^{1}$ \\ and François Feuillebois ${ }^{3} \dagger$ \\ ${ }^{1}$ Laboratoire Ingénierie Mathématique, École Polytechnique de Tunisie, rue El Khawarezmi, \\ BP 743, La Marsa, Tunisia \\ ${ }^{2}$ LadHyX, École Polytechnique, 91128 Palaiseau CEDEX, France \\ ${ }^{3}$ LIMSI-CNRS, UPR 3251, BP 133, 91403 Orsay CEDEX, France
}

(Received 23 November 2011; revised 4 July 2012; accepted 14 September 2012; first published online 23 October 2012)

The flow field around a solid particle moving in a shear flow along a porous slab is obtained by solving the coupled Stokes-Darcy problem with the Beavers and Joseph slip boundary condition on the slab interfaces. The solution involves the Green's function of this coupled problem, which is given here. It is shown that the classical boundary integral method using this Green's function is inappropriate because of supplementary contributions due to the slip on the slab interfaces. An 'indirect boundary integral method' is therefore proposed, in which the unknown density on the particle surface is not the actual stress, but yet allows calculation of the force and torque on the particle. Various results are provided for the normalized force and torque, namely friction factors, on the particle. The cases of a sphere and an ellipsoid are considered. It is shown that the relationships between friction coefficients (torque due to rotation and force due to translation) that are classical for a no-slip plane do not apply here. This difference is exhibited. Finally, results for the velocity of a freely moving particle in a linear and a quadratic shear flow are presented, for both a sphere and an ellipsoid.

Key words: boundary integral methods, low-Reynolds-number flows, porous media

\section{Introduction}

Modelling the motion of particles in a flow along a porous membrane has various practical applications. In cross-flow microfiltration (see Belfort, Davis \& Zydney 1994), suspended particles are retained by microporous membranes. The flow along the membrane is applied so as to diminish membrane clogging. In the asymmetric field-flow fractionation method in analytical chemistry (see Wahlund \& Giddings 1987), a transverse flow across a porous wall of a channel moves particles across the channel, so that they reach streamlines with different velocities and are thereby separated. In biology, cells entrained in a flow along a biological membrane may exchange chemicals across the membrane (see e.g. Khakpour \& Vafai 2008). 
More generally, we consider here a porous plane slab, the thickness of which is arbitrary, from a thin membrane up to a semi-infinite porous medium. The present paper is concerned with the model of a solid particle entrained in a shear flow along the porous slab. Typical particle dimensions, for example, for cross-flow microfiltration, are $0.01-100 \mu \mathrm{m}$ and typical pore sizes are $0.1-10 \mu \mathrm{m}$. Here we treat situations where the particle size is larger than the pore size, so that particles are trapped at the solid wall when the fluid is sucked across it.

Owing to the small size of particles suspended in water or in a fluid of similar viscosity, the Reynolds number relative to a particle is usually low, so that the Stokes equations of fluid motion apply. Here, Darcy's equations are used to model the flow in the porous medium with permeability $K>0$ adjacent to the fluid domain. As for values of the parameters, for Darcy's model to be valid the slab thickness $e$ should be much larger than the pore size, which is of the order of $\sqrt{K}$. Also, the particle size $a$ should be much larger than the pore size, $a \gg \sqrt{K}$. The selected ranges of these and additional parameters will be specified later on. Note that if $e / \sqrt{K}$ were either of order unity or smaller, Darcy's model would be insufficient and should be replaced by Brinkman's model, which better describes the fluid flow in pores. That model falls outside of the scope of the present article.

Continuity conditions for the flow normal velocity and pressure apply across the boundary between the fluid domain and the porous medium. As for the fluid velocity along the boundary, various models have been proposed. Classically, a no-slip condition is used on the fluid side, at scales that are large compared with the pore size. On the basis of their experiments, Beavers \& Joseph (1967) proposed to account for a slip velocity that is proportional to the shear rate at the scale of a pore:

$$
\frac{\partial \boldsymbol{u}_{s}}{\partial n}=\frac{\sigma}{\sqrt{K}}\left(\boldsymbol{u}_{s}-\boldsymbol{u}_{d}\right) .
$$

Here $\partial / \partial n$ is the derivative in the direction normal to the interface (where the $\boldsymbol{n}$ axis points into the fluid domain), and $\boldsymbol{u}_{s}$ and $\boldsymbol{u}_{d}$ are respectively the tangential component of the fluid velocity in the fluid flow and porous medium. The above slip depends on a dimensionless coefficient $\sigma \geqslant 0$ (1/ $\sigma$ may be called a slip coefficient) that is of order unity, typically in the range [0,10]. Richardson (1971), Taylor (1971) and Beavers, Sparrow \& Masha (1974) have shown that $\sigma$ strongly depends on the geometry of the transition region between the fluid and porous medium. Since $\sigma$ is of order unity, (1.1) then shows that the typical slip length $b=\sqrt{K} / \sigma$ is of the order of $\sqrt{K}$, that is, of the order of the pore size. The condition (1.1) was later formalized by Saffman (1971). However, he considered the case of a low permeability, keeping only the $\boldsymbol{u}_{s}$ term on the fluid side in (1.1) since $\boldsymbol{u}_{d}$ then is smaller. Other types of boundary conditions have been proposed; for example, Ochoa-Tapia \& Whitaker $(1995 a, b)$ consider a jump in the shear rate at the interface. This condition was later discussed in Chandesris \& Jamet (2007) and Valdés-Parada, Goyeau \& Ochoa-tapia (2007). Without entering the ongoing discussion about various possible conditions, the so-called Beavers and Joseph boundary condition (1.1), to be denoted hereafter as BJ, will be adopted here.

There have been various solutions for the problem of a particle near a porous slab in creeping flow. Goren (1979) provided an analytical solution for the motion of a sphere normal to a very thin porous membrane. He assumed the no-slip tangential condition on the porous medium interface. The departure of a sphere from contact with the membrane was treated by Nir (1981). Then, Sherwood (1988) solved the case 
of a sphere moving towards a permeable half-space, and Michalopoulou, Burganos \& Payatakes (1992) considered a solid sphere moving towards a permeable spherical obstacle. Note that Payatakes \& Dassios (1987) and Burganos et al. (1992) considered the opposite case of a permeable sphere moving towards a solid wall. Apart from these analytical solutions using bispherical coordinates (and tangent sphere coordinates for the contact situation), there have been numerical solutions in the case when the no-slip condition applies on the porous medium interface. Elasmi \& Feuillebois (2003) used the boundary integral method together with the Green's function that they calculated (see Elasmi \& Feuillebois 2001) to treat the case of a sphere moving towards a porous slab of finite thickness. The pressure was assumed to be constant on the other side of the slab. The method of fundamental solution was applied in Debbech, Elasmi \& Feuillebois (2010) to treat the case of a sphere moving towards a thin porous membrane, also using the Green's function obtained by Elasmi \& Feuillebois (2001).

This paper considers the motion of a solid and arbitrarily shaped particle entrained in a shear flow along a porous slab (there is also a transverse flow through the slab). By linearity of the Stokes and Darcy equations, this general problem may be considered as the sum of elementary ones. The tangential boundary condition on both interfaces of the slab is the slip condition (1.1). As opposed to the case treated in Elasmi \& Feuillebois (2003), there is no assumption concerning the flow on the other side of the slab, but more generally a condition at infinity in that fluid domain.

The first idea is to use a boundary integral method (BIM), which appears to be appropriate for such problems. For that purpose, an appropriate Green's function is derived. However, it is shown below that the classical BIM fails to represent the slip boundary condition on the porous interface. Therefore, an indirect boundary integral method (IBIM) is proposed instead.

The outline of the paper is as follows. Section 2 presents the problem at hand, together with the notation and basic material. It is then shown in $\S 3$ why the classical BIM raises a peculiar problem when using the slip boundary condition on a porous slab. The proposed novel IBIM avoids this difficulty. It eventually amounts to solving a Fredholm integral equation. The procedure to solve the integral equation numerically in the general case of a particle at any distance from the porous slab is presented in $\S 4$. Results are displayed and discussed in $\S 5$. Finally, conclusions are given in $\S 6$.

\section{Governing problems}

This section introduces the governing equations for the basic problems associated with the motion of a solid particle near a solid slab.

\subsection{Prescribed motion of a solid particle in a quiescent fluid}

Let us consider, as sketched in figure 1 , a motionless porous slab $\mathscr{S}$ with thickness $e$ and parallel plane boundaries $\Sigma_{l}$ and $\Sigma_{u}$ immersed in a Newtonian fluid with viscosity $\mu$ and density $\rho$.

In a Cartesian coordinate system $\left(x_{1}, x_{2}, x_{3}\right)$ with origin $O$ attached to the slab, the boundaries $\Sigma_{l}$ and $\Sigma_{u}$ are represented by $x_{3}=-e$ and $x_{3}=0$, respectively. A solid particle $\mathscr{P}$ with centre of mass $O^{\prime}$ and smooth boundary $S$ is immersed near $O$ in the upper $\left(x_{3}>0\right)$ fluid domain $\mathscr{D}$. Let the position of $O^{\prime}$ be on the $x_{3}$ axis and let $d$ be the gap between the particle and $\Sigma_{u}$. In this subsection, we consider a particle moving 


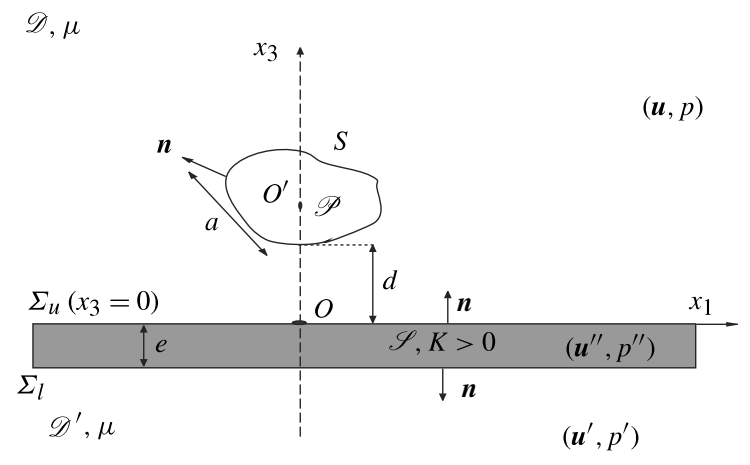

FIgURE 1. A solid particle $\mathscr{P}$ with length scale $a$ experiencing a prescribed rigid-body motion $(\boldsymbol{U}, \boldsymbol{\Omega})$ in a quiescent Newtonian fluid with viscosity $\mu$ above a porous slab $\mathscr{S}$ with permeability $K>0$.

in a quiescent fluid as a rigid body with translational velocity $\boldsymbol{U}$ (the velocity of $O^{\prime}$ ) and rotational velocity $\Omega$ relative to the slab. Such a motion induces flow fields in the domain $\mathscr{D}$, in the porous slab $\mathscr{S}$ and also in the lower fluid domain $\mathscr{D}^{\prime}$ represented by $x_{3} \leqslant-e$. Let the fluid velocity and pressure in domains $\mathscr{D}, \mathscr{D}^{\prime}$ and $\mathscr{S}$ be denoted by $(\boldsymbol{u}, p),\left(\boldsymbol{u}^{\prime}, p^{\prime}\right)$ and $\left(\boldsymbol{u}^{\prime \prime}, p^{\prime \prime}\right)$, respectively. Let the particle typical length scale be $a$ (for a spherical particle, $a$ will be its radius) and let the typical velocity scale on the particle surface be $V$. Assuming the Reynolds number to be low, i.e. $R e=\rho V a / \mu \ll 1$, the Stokes equations apply in the fluid domains:

$$
\begin{aligned}
& \mu \nabla^{2} \boldsymbol{u}=\nabla p, \quad \nabla \cdot \boldsymbol{u}=0 \quad \text { in } \mathscr{D}, \\
& \mu \nabla^{2} \boldsymbol{u}^{\prime}=\nabla p^{\prime}, \quad \nabla \cdot \boldsymbol{u}^{\prime}=0 \quad \text { in } \mathscr{D}^{\prime} .
\end{aligned}
$$

The flow in the porous slab $\mathscr{S}$ is modelled by the Darcy equations

$$
\boldsymbol{u}^{\prime \prime}=-\frac{K}{\mu} \nabla p^{\prime \prime}, \quad \nabla \cdot \boldsymbol{u}^{\prime \prime}=0 \quad \text { in } \mathscr{S},
$$

where $K \geqslant 0$ is the permeability of the porous medium. Equations (2.1) and (2.2) are supplemented by several boundary conditions. At the porous slab boundaries, continuity conditions apply for the pressure and the normal component of the velocity:

$$
\begin{array}{ccl}
p^{\prime}=p^{\prime \prime}, & \left(\boldsymbol{u}^{\prime}-\boldsymbol{u}^{\prime \prime}\right) \cdot \boldsymbol{e}_{3}=0 & \text { on } \Sigma_{l}, \\
p=p^{\prime \prime}, & \left(\boldsymbol{u}-\boldsymbol{u}^{\prime \prime}\right) \cdot \boldsymbol{e}_{3}=0 & \text { on } \Sigma_{u},
\end{array}
$$

where $\boldsymbol{e}_{3}$ is the unit normal vector in the $x_{3}$ direction. In the tangential direction, the BJ boundary condition (1.1) is assumed to apply on both boundaries:

$$
\begin{gathered}
\frac{\partial \boldsymbol{u}^{\prime}}{\partial x_{3}} \cdot \boldsymbol{e}_{j}=-\frac{\sigma}{\sqrt{K}}\left(\boldsymbol{u}^{\prime}-\boldsymbol{u}^{\prime \prime}\right) \cdot \boldsymbol{e}_{j} \quad(\text { for } j=1,2) \text { on } \Sigma_{l}, \\
\frac{\partial \boldsymbol{u}}{\partial x_{3}} \cdot \boldsymbol{e}_{j}=\frac{\sigma}{\sqrt{K}}\left(\boldsymbol{u}-\boldsymbol{u}^{\prime \prime}\right) \cdot \boldsymbol{e}_{j} \quad(\text { for } j=1,2) \text { on } \Sigma_{u},
\end{gathered}
$$

where $\boldsymbol{e}_{j}$ is the unit normal vector in direction $j=1,2$. The boundary conditions (2.4) then allow for a slip of the fluid on the porous slab boundaries. 
In this subsection, we consider that the fluid is at rest far from the moving particle, and thus the far-field behaviour is

$$
\left(\boldsymbol{u}, \boldsymbol{u}^{\prime}, \boldsymbol{u}^{\prime \prime}\right) \rightarrow(\mathbf{0}, \mathbf{0}, \mathbf{0}), \quad\left(p, p^{\prime}, p^{\prime \prime}\right) \rightarrow(0,0,0) \quad \text { as }|\boldsymbol{x}| \rightarrow \infty
$$

where $\boldsymbol{x}$ is a vector pointing from $O$ to a point in the flow field. Finally, the no-slip boundary condition on the particle surface is, setting $\boldsymbol{x}^{\prime}=\boldsymbol{x}-\boldsymbol{O} \boldsymbol{O}^{\prime}$,

$$
\boldsymbol{u}=\boldsymbol{U}+\boldsymbol{\Omega} \times \boldsymbol{x}^{\prime} \text { on } S .
$$

The flow field $(\boldsymbol{u}, p)$ with stress tensor $\boldsymbol{\sigma}$ exerts a force $\boldsymbol{F}_{h}$ on the particle and a torque $\Gamma_{h}$ about its centre of mass $O^{\prime}$ given by

$$
\boldsymbol{F}_{h}=\int_{S} \boldsymbol{\sigma} \cdot \boldsymbol{n} \mathrm{d} S, \quad \boldsymbol{\Gamma}_{h}=\int_{S} \boldsymbol{x}^{\prime} \times \boldsymbol{\sigma} \cdot \boldsymbol{n} \mathrm{d} S,
$$

where $\boldsymbol{n}$ is the unit vector normal to $S$ and pointing into the fluid. For a prescribed particle $\mathscr{P}$ (i.e. prescribed shape and location) and rigid-body motion $(\boldsymbol{U}, \boldsymbol{\Omega})$, the coupled flow fields $(\boldsymbol{u}, p),\left(\boldsymbol{u}^{\prime}, p^{\prime}\right)$ and $\left(\boldsymbol{u}^{\prime \prime}, p^{\prime \prime}\right)$ solutions of (2.1)-(2.6) and the vectors $\boldsymbol{F}_{h}$ and $\boldsymbol{\Gamma}_{h}$ depend upon the lengths $e, \sqrt{K}$ and the dimensionless parameter $\sigma$.

We end this subsection with some classical material and notation. By linearity of the force and torque functionals, the force and torque (2.7) are obtained in the form

$$
\boldsymbol{F}_{h}=-\mu[\boldsymbol{A} \cdot \boldsymbol{U}+\boldsymbol{B} \cdot \boldsymbol{\Omega}], \quad \boldsymbol{\Gamma}_{h}=-\mu[\boldsymbol{C} \cdot \boldsymbol{U}+\boldsymbol{D} \cdot \boldsymbol{\Omega}],
$$

where $\boldsymbol{A}, \boldsymbol{B}, \boldsymbol{C}$ and $\boldsymbol{D}$ are second-rank tensors. It is then equivalent to our problem and practically simpler to search, by linearity, for the sum of six independent flow fields in $\mathscr{D}$, three for translation $\left(\boldsymbol{u}_{t}^{(i)}, p_{t}^{(i)}\right)$ and three for rotation $\left(\boldsymbol{u}_{r}^{(i)}, p_{r}^{(i)}\right)$, for $i=1,2,3$, with the following boundary conditions on the solid particle:

$$
\boldsymbol{u}_{t}^{(i)}=\boldsymbol{e}_{i} \quad \text { and } \quad \boldsymbol{u}_{r}^{(i)}=\boldsymbol{e}_{i} \times \boldsymbol{x}^{\prime} \quad(\text { for } i=1,2,3) \text { on } S
$$

and related flow fields in $\mathscr{S}$ and $\mathscr{D}^{\prime}$, solutions of (2.1)-(2.5). Denoting the associated stress tensors by $\boldsymbol{\sigma}_{t}^{(i)}$ and $\boldsymbol{\sigma}_{r}^{(i)}$, the Cartesian components of the tensors $\boldsymbol{A}, \boldsymbol{B}, \boldsymbol{C}$ and $\boldsymbol{D}$ introduced in (2.8) therefore obey:

$$
\begin{array}{cc}
A_{j i}=-\frac{1}{\mu} \int_{S}\left[\boldsymbol{\sigma}_{t}^{(i)} \cdot \boldsymbol{n}\right] \cdot \boldsymbol{e}_{j} \mathrm{~d} S, \quad B_{j i}=-\frac{1}{\mu} \int_{S}\left[\boldsymbol{\sigma}_{r}^{(i)} \cdot \boldsymbol{n}\right] \cdot \boldsymbol{e}_{j} \mathrm{~d} S, \\
C_{j i}=-\frac{1}{\mu} \int_{S}\left[\boldsymbol{x}^{\prime} \times \boldsymbol{\sigma}_{t}^{(i)} \cdot \boldsymbol{n}\right] \cdot \boldsymbol{e}_{j} \mathrm{~d} S, & D_{j i}=-\frac{1}{\mu} \int_{S}\left[\boldsymbol{x}^{\prime} \times \boldsymbol{\sigma}_{r}^{(i)} \cdot \boldsymbol{n}\right] \cdot \boldsymbol{e}_{j} \mathrm{~d} S .
\end{array}
$$

For $K=0$, the problem reduces to a fluid motion along a no-slip wall $\Sigma_{u}$ (as $\sigma / \sqrt{K} \rightarrow \infty)$ and the Lorentz reciprocity theorem provides the classical result that $\boldsymbol{B}$ and $\boldsymbol{C}$ are one another's transpose. Whenever $K>0$, this property breaks down as detailed below in $\S 2.3$.

\subsection{Migration of a solid particle immersed in a prescribed ambient flow field and/or a gravity field}

Consider now the case of a solid particle either held fixed in an ambient flow field or freely suspended in a flow field and/or a gravity field. These circumstances are illustrated in figure 2.

We consider a class of elementary ambient flow fields $\left(\boldsymbol{u}_{\infty}, p_{\infty}\right)$ in $\mathscr{D}$ (with associated flow fields $\left(\boldsymbol{u}_{\infty}^{\prime}, p_{\infty}^{\prime}\right)$ in $\mathscr{D}^{\prime}$ and $\left(\boldsymbol{u}_{\infty}^{\prime \prime}, p_{\infty}^{\prime \prime}\right)$ in $\left.\mathscr{S}\right)$ providing a good 
(a)

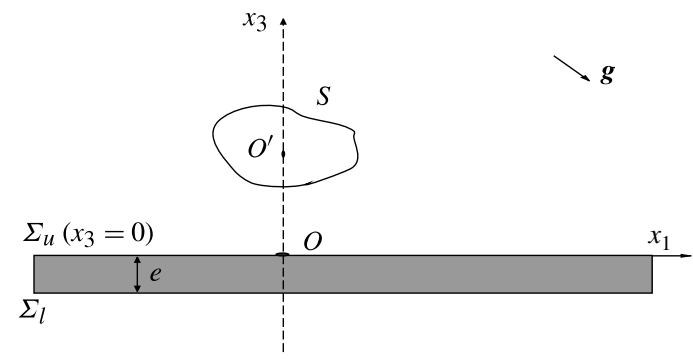

(b)

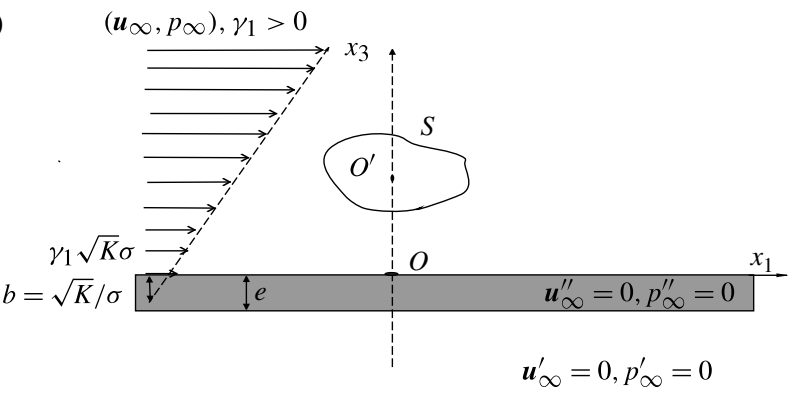

(c)

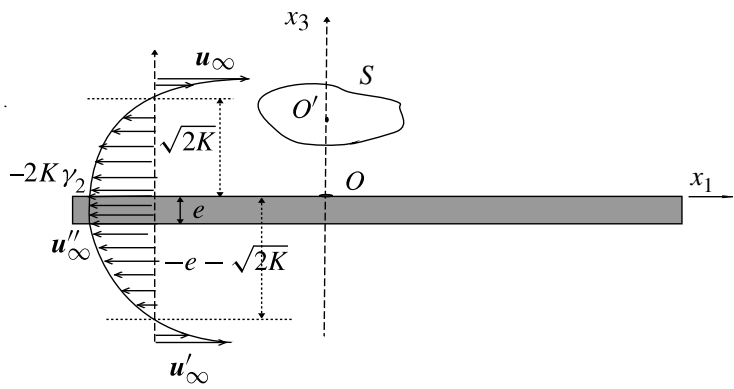

FIGURE 2. A solid particle suspended in the ambient flow field $\left(\boldsymbol{u}_{\infty}, p_{\infty}\right)$ and/or uniform gravity field $\boldsymbol{g}$. (a) Case $\left(\boldsymbol{u}_{\infty}, p_{\infty}\right)=(\mathbf{0}, 0)$. (b) Case $\boldsymbol{g}=\mathbf{0}, \gamma_{2}=0$ (see (2.13)) and $\gamma_{1}>0$. There is no flow in the lower domain $\left(x_{3}<-e\right)$ and in the slab. (c) Ambient velocity profiles for $\boldsymbol{g}=\mathbf{0}, \gamma_{1}=0$ and $\gamma_{2}>0$ (see $(2.13 a)$ ). Here, flows also occur in the porous slab and in the lower fluid domain.

approximation of a Navier-Stokes flow field near the porous slab when in microfiltration. With this goal in mind, we take in $\mathscr{D}$ the following ambient flow field in which the velocity varies as a second-degree polynomial in the $x_{3}$ coordinate:

$$
\boldsymbol{u}_{\infty}=\left(\gamma_{0}+\gamma_{1} x_{3}+\gamma_{2} x_{3}^{2}\right) \boldsymbol{e}_{1}+A \boldsymbol{e}_{3}, \quad p_{\infty}=2 \mu \gamma_{2} x_{1} .
$$

These equations represent a pressure-driven flow parallel to the slab plus a uniform flow normal to it. The detailed associated flow fields $\left(\boldsymbol{u}_{\infty}^{\prime}, p_{\infty}^{\prime}\right)$ and $\left(\boldsymbol{u}_{\infty}^{\prime \prime}, p_{\infty}^{\prime \prime}\right)$ are obtained using (2.1)-(2.4). The $\gamma_{0}$ term in (2.11) allows for a uniform slip along the slab, whereas $\gamma_{1}$ and $\gamma_{2}$ allow respectively for linear and quadratic shear flows parallel to it. Finally, the normal flow velocity $A \boldsymbol{e}_{3}$ in (2.11) is a permeation flow across the slab. In the absence of parallel flow, one obtains $\boldsymbol{u}_{\infty}=A \boldsymbol{e}_{3}$ in $\mathscr{D}, \mathscr{S}$ and $\mathscr{D}^{\prime}$. In this case, the flow field with a fixed particle is obtained from the one due to a particle moving with velocity $\boldsymbol{U}=-\boldsymbol{A e}_{3}$ in a quiescent fluid. It is therefore tractable using the 
results of $\S 2.1$. It is then sufficient to restrict our attention in this subsection to the ambient unidirectional flow field:

$$
\boldsymbol{u}_{\infty}=\left(\gamma_{0}+\gamma_{1} x_{3}+\gamma_{2} x_{3}^{2}\right) \boldsymbol{e}_{1}, \quad p_{\infty}=2 \mu \gamma_{2} x_{1} .
$$

Enforcing (2.1)-(2.4) actually requires $\gamma_{0}$ to depend linearly upon $\gamma_{1}$ and $\gamma_{2}$. Curtailing elementary manipulations, the ambient flow fields in each domain then read:

$$
\begin{gathered}
\boldsymbol{u}_{\infty}=\gamma_{1}\left[x_{3}+\sqrt{K} / \sigma\right] \boldsymbol{e}_{1}+\gamma_{2}\left[x_{3}^{2}-2 K\right] \boldsymbol{e}_{1}, \quad p_{\infty}=2 \mu \gamma_{2} x_{1} \quad \text { for } x_{3} \geqslant 0, \\
\boldsymbol{u}_{\infty}^{\prime}=\gamma_{1}^{\prime}\left[x_{3}+e-\sqrt{K} / \sigma\right] \boldsymbol{e}_{1}+\gamma_{2}\left[\left(x_{3}+e\right)^{2}-2 K\right] \boldsymbol{e}_{1}, \\
p_{\infty}^{\prime}=2 \mu \gamma_{2} x_{1} \quad \text { for } x_{3} \leqslant-e, \\
\boldsymbol{u}_{\infty}^{\prime \prime}=-2 \gamma_{2} K \boldsymbol{e}_{1}, \quad p_{\infty}^{\prime \prime}=2 \mu \gamma_{2} x_{1} \quad \text { for }-e \leqslant x_{3} \leqslant 0 .
\end{gathered}
$$

Observe that the additional coefficient $\gamma_{1}^{\prime}$ in $(2.13 b)$ is not related to $\gamma_{1}$. Indeed, for $\gamma_{2}=0$, the pressure and velocity in the porous slab vanish and the flows in the upper and lower domains $\mathscr{D}$ and $\mathscr{D}^{\prime}$ then uncouple. For the filtration problem where a flow field is imposed in $\mathscr{D}$, the shear rate $\gamma_{1}^{\prime}$ in $\mathscr{D}^{\prime}$ is undefined and thus irrelevant. It may as well be taken as zero, as illustrated in figure $2(b)$. Note that, because of the pressure gradient in $\mathscr{D}^{\prime}$, formally the velocity is growing for $x_{3} \rightarrow-\infty$. Practically, only the velocity in the region close to the slab is of interest in $\mathscr{D}^{\prime}$ and it is expected that the velocity along the slab is low.

When the solid particle $\mathscr{P}$ is held fixed in the undisturbed flow field $\left(\boldsymbol{u}_{\infty}, p_{\infty}\right)$, the disturbed flow fields may be expressed as $\left(\boldsymbol{u}_{\infty}+\boldsymbol{u}, p_{\infty}+p\right)$ in $\mathscr{D},\left(\boldsymbol{u}_{\infty}^{\prime}+\right.$ $\left.\boldsymbol{u}^{\prime}, p_{\infty}^{\prime}+p^{\prime}\right)$ in $\mathscr{D}^{\prime}$ and $\left(u_{\infty}^{\prime \prime}+\boldsymbol{u}^{\prime \prime}, p_{\infty}^{\prime \prime}+p^{\prime \prime}\right)$ in $\mathscr{S}$. The disturbance flows $(\boldsymbol{u}, p)$, $\left(\boldsymbol{u}^{\prime}, p^{\prime}\right)$ and $\left(\boldsymbol{u}^{\prime \prime}, p^{\prime \prime}\right)$ vanish far from the particle and should by linearity satisfy (2.1)-(2.5). Finally, the no-slip condition on the motionless solid particle requires that

$$
\boldsymbol{u}=-\boldsymbol{u}_{\infty} \quad \text { on } S .
$$

Solving (2.1)-(2.5) in conjunction with (2.14) provides the flow field $(\boldsymbol{u}, p)$, its stress tensor $\boldsymbol{\sigma}$ and the surface stress $\boldsymbol{f}=\boldsymbol{\sigma} \cdot \boldsymbol{n}$ exerted on the particle. The undisturbed flow field $\left(\boldsymbol{u}_{\infty}, p_{\infty}\right)$ in domain $\mathscr{D}$ exerts zero force and torque on the particle. Accordingly, the particle experiences in the disturbed flow $\left(\boldsymbol{u}_{\infty}+\boldsymbol{u}, p_{\infty}+p\right)$ a force $\boldsymbol{F}_{e}$ and torque $\Gamma_{e}$ about $O^{\prime}$ given by

$$
\boldsymbol{F}_{e}=\int_{S} \boldsymbol{f} \mathrm{d} S, \quad \boldsymbol{\Gamma}_{e}=\int_{S} \boldsymbol{x}^{\prime} \times \boldsymbol{f} \mathrm{d} S .
$$

Superimposing a uniform gravity field $\boldsymbol{g}$ solely adds to each ambient pressure field the hydrostatic term $\rho \boldsymbol{g} \cdot \boldsymbol{x}$, where $\rho$ is the fluid mass density. When immersed in the ambient flow field $\left(\boldsymbol{u}_{\infty}, p_{\infty}\right)$ and gravity field $\boldsymbol{g}$, a solid particle with centre of mass $O^{\prime}$, volume $\mathscr{V}$ and uniform density $\rho_{s}$ in rigid-body motion $(\boldsymbol{U}, \boldsymbol{\Omega})$ then experiences a net force $\boldsymbol{F}$ and net torque $\boldsymbol{\Gamma}$ about $O^{\prime}$ given by

$$
\begin{aligned}
& \boldsymbol{F}=\boldsymbol{F}_{e}+\boldsymbol{F}_{h}+\left(\rho_{s}-\rho\right) \mathscr{V} \boldsymbol{g}, \\
& \boldsymbol{\Gamma}=\boldsymbol{\Gamma}_{e}+\boldsymbol{\Gamma}_{h} .
\end{aligned}
$$

Depending upon the application, any other physical force and torque (e.g. magnetic force and torque when in a magnetic field) may simply be added on the right-hand side of $(2.16 a)$ and $(2.16 b)$, respectively. 
For a freely suspended particle with negligible inertia, the conditions $\boldsymbol{F}=\boldsymbol{\Gamma}=\mathbf{0}$ provide the linear system

$$
\begin{aligned}
& \mu(\boldsymbol{A} \cdot \boldsymbol{U}+\boldsymbol{B} \cdot \boldsymbol{\Omega})=\boldsymbol{F}_{e}+\left(\rho_{s}-\rho\right) \mathscr{V} \boldsymbol{g}, \\
& \mu(\boldsymbol{C} \cdot \boldsymbol{U}+\boldsymbol{D} \cdot \boldsymbol{\Omega})=\boldsymbol{\Gamma}_{e},
\end{aligned}
$$

which is to be solved for the unknown rigid-body migration $(U, \Omega)$.

\subsection{Summary and basic remarks}

As shown in $\S \S 2.1$ and 2.2, it is sufficient to solve the problem (2.1)-(2.5), on one side with (2.9) for the six auxiliary flow fields $\left(\boldsymbol{u}_{t}^{(i)}, p_{t}^{(i)}\right)$ and $\left(\boldsymbol{u}_{r}^{(i)}, p_{r}^{(i)}\right)(i=1,2,3)$, and on the other side with (2.14) for two specific ambient flows $\left(\boldsymbol{u}_{\infty}^{1}, p_{\infty}^{1}\right)$ and $\left(\boldsymbol{u}_{\infty}^{2}, p_{\infty}^{2}\right)$ given by $(2.13 a)$ with $\left(\gamma_{1}, \gamma_{2}\right)=(1,0)$ and $(0,1)$, respectively.

Using the reciprocal identity for two Stokes flows that are vanishing far from the particle in the upper fluid domain $\mathscr{D}$ yields for the second-rank tensors $\boldsymbol{B}$ and $\boldsymbol{C}$ the relationship

$$
B_{j i}-C_{i j}=\frac{1}{\mu} \int_{\Sigma_{u}}\left[\boldsymbol{u}_{t}^{(j)} \cdot \boldsymbol{\sigma}_{r}^{(i)}-\boldsymbol{u}_{r}^{(i)} \cdot \boldsymbol{\sigma}_{t}^{(j)}\right] \cdot \boldsymbol{e}_{3} \mathrm{~d} S .
$$

A similar relationship for the flow disturbance $(\boldsymbol{u}, p)$ governed by (2.1)-(2.5) and (2.14), as introduced in $\S 2.2$, yields the expression of the force (with a summation over indices $i$ in (2.19)) as

$$
\boldsymbol{F}_{e}=\left\{-\int_{S} \boldsymbol{u}_{\infty} \cdot \boldsymbol{f}_{t}^{(i)} \mathrm{d} S+\int_{\Sigma_{u}}\left[\boldsymbol{u} \cdot \boldsymbol{\sigma}_{t}^{(i)}-\boldsymbol{u}_{t}^{(i)} \cdot \boldsymbol{\sigma}\right] \cdot \boldsymbol{e}_{3}\right\} \cdot \boldsymbol{e}_{i} .
$$

An expression for the torque $\boldsymbol{\Gamma}_{e}$ is obtained by replacing the subscript $t$ with $r$ in the right-hand side of (2.19). In the standard case of a solid no-slip plane $\Sigma_{u}$ (case $K=0)$, the integrals over $\Sigma_{u}((2.18)$ and (2.19)) vanish and the expressions simplify. For $K>0$ there is a priori no reason for this property to be true any more, because of the quite different boundary conditions $(2.3 b)$ and $(2.4 b)$ on the upper boundary $\Sigma_{u}$ of the porous slab. Indeed, the numerical results presented later in figure 5 clearly show that $B_{j i} \neq C_{i j}$ for an example case. Moreover, evaluating $\boldsymbol{F}_{e}$ (or $\boldsymbol{\Gamma}_{e}$ ) using (2.19) would unfortunately require the determination of the velocities $\boldsymbol{u}, \boldsymbol{u}_{t}^{(i)}$ and $\boldsymbol{u}_{r}^{(i)}$ and associated stresses $(\boldsymbol{\sigma} \cdot \boldsymbol{n}),\left(\boldsymbol{\sigma}_{t}^{(i)} \cdot \boldsymbol{n}\right)$ and $\left(\boldsymbol{\sigma}_{r}^{(i)} \cdot \boldsymbol{n}\right)$ on the entire unbounded surface $\boldsymbol{\Sigma}_{u}$.

It thus appears highly preferable to look for a different formulation that solely involves integrals over the particle surface $S$, like in the original expressions (2.15). This challenging issue is treated in $\S 3$.

\section{A proposed indirect boundary integral formulation}

Recall that the problem, as presented in $\S 2$, consists of the Stokes flow field $(\boldsymbol{u}, p)$ in the upper fluid domain $\mathscr{D}$ with associated Stokes flow field $\left(\boldsymbol{u}^{\prime}, p^{\prime}\right)$ in the lower fluid domain $\mathscr{D}^{\prime}$ and the Darcy flow field $\left(\boldsymbol{u}^{\prime \prime}, p^{\prime \prime}\right)$ in the porous slab $\mathscr{S}$. These coupled flows should satisfy (2.1)-(2.5) with, depending on the addressed problem, either boundary condition (2.9) or (2.14).

The idea is to use a boundary integral formulation on the basis of the Green's tensor of the problem. It will be first shown in $\S 3.1$ that the classical boundary integral method (BIM) is inappropriate. The formulation of an indirect boundary integral method (IBIM) will then be presented. Details of the Green's tensor will be given in $\S 3.2$. 


\subsection{Green's function and the indirect formulation}

Henceforth, we use the usual tensor summation. The essential ingredient for the boundary integral formulation is the Green's function, say $\boldsymbol{V}^{(k)}(\boldsymbol{x}, \boldsymbol{y})$ and $P^{(k)}(\boldsymbol{x}, \boldsymbol{y})$ for the fluid velocity and pressure at point $\boldsymbol{x}$ in the $x_{3}>0$ domain, due to a unit point force $f \boldsymbol{e}_{k}$ in the $k$ direction that is applied at point $\boldsymbol{y}$ in the $y_{3}>0$ domain. By definition, the velocity and pressure are solutions of

$$
\boldsymbol{\nabla} \cdot \boldsymbol{V}^{(k)}(\boldsymbol{x}, \boldsymbol{y})=0, \quad \mu \nabla^{2} \boldsymbol{V}^{(k)}(\boldsymbol{x}, \boldsymbol{y})-\nabla P^{(k)}(\boldsymbol{x}, \boldsymbol{y})+f \delta(\boldsymbol{x}-\boldsymbol{y}) \boldsymbol{e}_{k}=0 \quad \text { for } x_{3}>0,
$$

where $\delta$ is the three-dimensional Dirac delta function. Equations (3.1) are coupled with the associated Darcy equations (2.2) in the porous slab $\mathscr{S}$ and the Stokes equations $(2.1 b)$ in the lower fluid domain $\mathscr{D}^{\prime}$, with relevant boundary conditions between domains (2.3) and (2.4) and conditions at infinity (2.5).

The solution was calculated analytically by Khabthani (2004). The fluid velocity component $j$ was obtained in the form

$$
\boldsymbol{V}^{(k)}(\boldsymbol{x}, \boldsymbol{y}) \cdot \boldsymbol{e}_{j}=\frac{f}{8 \pi \mu} G_{j k}(\boldsymbol{x}, \boldsymbol{y})=\frac{f}{8 \pi \mu}\left[G_{j k}^{\infty}(\boldsymbol{x}, \boldsymbol{y})+R_{j k}(\boldsymbol{x}, \boldsymbol{y})\right],
$$

where the first part $(f / 8 \pi \mu) G_{j k}^{\infty}$ is the classical free-space Green's tensor, or stokeslet, with the Oseen tensor

$$
G_{j k}^{\infty}(\boldsymbol{x}, \boldsymbol{y})=\frac{\delta_{j k}}{|\boldsymbol{x}-\boldsymbol{y}|}+\frac{\left[(\boldsymbol{x}-\boldsymbol{y}) \cdot \boldsymbol{e}_{j}\right]\left[(\boldsymbol{x}-\boldsymbol{y}) \cdot \boldsymbol{e}_{k}\right]}{|\boldsymbol{x}-\boldsymbol{y}|^{3}},
$$

where $\delta_{j k}$ is the Kronecker delta. The second term $R_{j k}(\boldsymbol{x}, \boldsymbol{y})$ in (3.2) stands for images of $G_{j k}^{\infty}(\boldsymbol{x}, \boldsymbol{y})$ in the porous slab. Unlike $G_{j k}^{\infty}$, the tensor $R_{j k}$ is smooth in the entire $x_{3}>0$ domain. Its expression in domain $\mathscr{D}$ is given below in $\S 3.2$. Some details of the derivation and the results in the whole space are given in appendix $\mathrm{B}$. One should note (see (2.18)) that here $G_{j k}(\boldsymbol{x}, \boldsymbol{y}) \neq G_{k j}(\boldsymbol{y}, \boldsymbol{x})$, i.e that the widely encountered symmetry property of the usual Green's tensors (see Pozrikidis 1992, chap. 4) does not hold in the present work. The associated pressure of the Green's function, $P^{(k)}(\boldsymbol{x}, \boldsymbol{y})$, is obtained as

$$
P^{(k)}(\boldsymbol{x}, \boldsymbol{y})(\boldsymbol{x}, \boldsymbol{y})=\frac{f}{8 \pi} H_{k}(\boldsymbol{x}, \boldsymbol{y})=\frac{f}{8 \pi}\left[H_{k}^{\infty}(\boldsymbol{x}, \boldsymbol{y})+Q_{k}(\boldsymbol{x}, \boldsymbol{y})\right],
$$

where $(f / 8 \pi \mu) H_{k}^{\infty}(\boldsymbol{x}, \boldsymbol{y})$ is the pressure associated with $(f / 8 \pi \mu) G_{j k}^{\infty}$, where

$$
H_{k}^{\infty}(\boldsymbol{x}, \boldsymbol{y})=\frac{2\left(x_{k}-y_{k}\right)}{|\boldsymbol{x}-\boldsymbol{y}|^{3}} .
$$

The second term $Q_{k}(\boldsymbol{x}, \boldsymbol{y})$ in (3.4) is associated with the images in the porous slab. This quantity, also obtained in Khabthani (2004), may be deduced from the material displayed in appendix B.

A basic consequence of the non-symmetry of $G_{j k}$ is that, when applying the classical approach to obtain a boundary integral formulation, the result for the perturbation fluid velocity $\boldsymbol{u}(\boldsymbol{x})$ is the following integral representation:

$$
u_{k}(\boldsymbol{x})=-\frac{1}{8 \pi \mu} \int_{S} \sigma_{i j}(\boldsymbol{y}) n_{j} G_{i k}(\boldsymbol{y}, \boldsymbol{x}) \mathrm{d} S(\boldsymbol{y})+C_{k}\left(\boldsymbol{x}, \Sigma_{u}\right) \quad \text { at } \boldsymbol{x} \in \mathscr{D},
$$

where $\boldsymbol{\sigma}(\boldsymbol{y})$ is the stress tensor (with Cartesian component $\sigma_{i j}$ ) associated with the unknown flow field at $\boldsymbol{y}$ on the particle surface $S$, and $\boldsymbol{n}$ is the normal unit vector on $S$ pointing into the fluid domain $\mathscr{D}$. The BJ condition implies that there is a left-over 
term $C_{k}\left(\boldsymbol{x}, \Sigma_{u}\right)$ on the upper side $\Sigma_{u}$ of the porous membrane. This term is given for $\boldsymbol{x} \in \mathscr{D}$ by

$$
C_{k}\left(\boldsymbol{x}, \Sigma_{u}\right)=-\frac{1}{8 \pi \mu} \int_{\Sigma_{u}}\left\{G_{i k}(\boldsymbol{y}, \boldsymbol{x}) \sigma_{i j}(\boldsymbol{y})-u_{i}(\boldsymbol{y}) T_{i k j}(\boldsymbol{y}, \boldsymbol{x})\right\} n_{j}(\boldsymbol{y}) \mathrm{d} S(\boldsymbol{y}),
$$

where the stress tensor associated with the Green's function $\boldsymbol{V}^{(k)}$ has Cartesian component $T_{i k j} /(8 \pi)$. In the case of the no-slip condition on $\Sigma_{u}$, this term was found to vanish by Elasmi \& Feuillebois (2003), but this is no longer the case here. This left-over integral on $\Sigma_{u}$ introduces complications in the application of the classical boundary integral method.

The main idea of the proposed integral formulation is then to express the unknown fluid velocity $\boldsymbol{u}(\boldsymbol{x})$ in terms of a single layer on the particle boundary. In other words, we write

$$
\boldsymbol{u}(\boldsymbol{x})=-\frac{1}{8 \pi \mu} \int_{S} \boldsymbol{G}(\boldsymbol{x}, \boldsymbol{y}) \cdot \boldsymbol{d}(\boldsymbol{y}) \mathrm{d} S \text { for } \boldsymbol{x} \text { in } \mathscr{D},
$$

where $\boldsymbol{d}$ is an unknown surface density of forces. Setting $\boldsymbol{H}=H_{k} \boldsymbol{e}_{k}$, the fluid pressure is obtained from the associated integral representation as

$$
p(\boldsymbol{x})=-\frac{1}{8 \pi} \int_{S} \boldsymbol{H}(\boldsymbol{x}, \boldsymbol{y}) \cdot \boldsymbol{d}(\boldsymbol{y}) \mathrm{d} S \text { for } \boldsymbol{x} \text { in } \mathscr{D} .
$$

Note that the fluid velocity and pressure in $\mathscr{S}$ and $\mathscr{D}^{\prime}$ may similarly be expressed as integrals on the particle boundary $S$, using the appropriate formulae for the Green's function $(\boldsymbol{G}, \boldsymbol{H})$ in those regions (see appendix B). Such expressions will automatically satisfy the boundary conditions between media $\mathscr{D}, \mathscr{S}$ and $\mathscr{D}^{\prime}$ (for instance, the slip velocity on the porous slab boundaries may then be calculated).

Since $\boldsymbol{G}^{\infty}$ and $\boldsymbol{G}$ are weakly singular as $\boldsymbol{x} \rightarrow \boldsymbol{y}$, the expression (3.8) still holds for $\boldsymbol{x}$ located on the particle surface $S$. On this surface, the fluid velocity takes a prescribed value, say $\boldsymbol{u}_{d}$, expressing either boundary condition (2.9) or (2.14). In the limit where $\boldsymbol{x}$ goes onto the sphere surface, (3.8) provides a Fredholm boundary integral equation of the first kind to be solved for the density $\boldsymbol{d}$ :

$$
-\frac{1}{8 \pi \mu} \int_{S} \boldsymbol{G}(\boldsymbol{x}, \boldsymbol{y}) \cdot \boldsymbol{d}(\boldsymbol{y}) \mathrm{d} S=\boldsymbol{u}_{d}(\boldsymbol{x}) \text { for } \boldsymbol{x} \text { on } S .
$$

It should be emphasized that the proposed formulation (3.8)-(3.10) is not just a 'blind' application of the classical integral formulation in unbounded space. This deserves a few comments.

(a) It solely involves an integral on the particle boundary $S$, i.e. not on the unbounded surface $\Sigma_{u}$. But it rests on the expression of the Green's tensor, which involves the whole flow field, including the boundary conditions on those surfaces.

(b) For a given flow field $(\boldsymbol{u}(\boldsymbol{x}), p(\boldsymbol{x}))$ in $\mathscr{D}$, the surface density $\boldsymbol{d}(\boldsymbol{y})$ on $S$ is uniquely determined, up to $p_{0} \boldsymbol{n}$, where $p_{0}$ is a constant and $\boldsymbol{n}$ is a unit vector normal to $S$ at point $\boldsymbol{y}$. This classical indeterminacy arises, like in BIM, since the pressure in incompressible flow is defined up to a constant $p_{0}$. The demonstration of this uniqueness (within $p_{0} \boldsymbol{n}$ ) is analogous to that in Elasmi \& Feuillebois (2003) (see $\S 4.3$ there).

(c) Unlike in the classical formulation, the density $\boldsymbol{d}$ has no physical meaning. For instance, it is not the surface stress $\boldsymbol{\sigma} \cdot \boldsymbol{n}$ exerted on $S$ by the flow field. For this reason, as announced above, the approach (3.8)-(3.10) is called here indirect. 
After numerically solving (3.10) for $\boldsymbol{d}$, the fluid velocity $\boldsymbol{u}$ in $\mathscr{D}$ may be obtained, if needed, from (3.8).

Like in the free-space case, $\nabla \boldsymbol{G}(\boldsymbol{x}, \boldsymbol{y})$ and $\boldsymbol{H}(\boldsymbol{x}, \boldsymbol{y})$ exhibit strongly singular behaviour of the order of $|\boldsymbol{x}-\boldsymbol{y}|^{-2}$ on the particle surface $S$ as $\boldsymbol{x} \rightarrow \boldsymbol{y}$. This property prevents one from using (3.8) and (3.9) to calculate the force and torque on the particle by integration of the stress and its moment on $S$. Another possible and efficient way is to extract the singular part of the Green's function by writing

$$
\boldsymbol{u}_{k}=\boldsymbol{u}_{k}^{\infty}+\boldsymbol{v}_{k}, \quad p_{k}=p_{k}^{\infty}+q_{k},
$$

in which the singular part is the unbounded flow Green's function $\left(\boldsymbol{u}_{k}^{\infty}, p_{k}^{\infty}\right)$ and the $\left(\boldsymbol{v}_{k}, q_{k}\right)$ flow is due to its images in $\mathscr{S}$. Only the singular part contributes to the force $\boldsymbol{F}$ and torque $\boldsymbol{\Gamma}$ on the particle. Exploiting Pozrikidis (1992) for this singular part, we obtain

$$
\boldsymbol{F}=\int_{S} \boldsymbol{d} \mathrm{d} S, \quad \boldsymbol{\Gamma}=\int_{S}\left(\boldsymbol{x}^{\prime} \times \boldsymbol{d}\right) \mathrm{d} S .
$$

That is, the density $\boldsymbol{d}$ (retained up to a constant multiple of $\boldsymbol{n}$ ), even though it is not the local stress $\boldsymbol{f}=\boldsymbol{\sigma} \cdot \boldsymbol{n}$, provides directly the required force and torque on the particle. Of course, determining other quantities related to $f$ such as the stresslet tensor would require $\boldsymbol{f}=\boldsymbol{\sigma} \cdot \boldsymbol{n}$ to be obtained on the surface $S$. As explained in appendix A, it is possible to achieve this task from the knowledge of $\boldsymbol{d}$ by (numerically) inverting on $S$ another boundary integral equation.

\subsection{Determination of the Green's tensor}

As presented above, the proposed procedure rests on the determination of the Green's tensor $G_{j k}(\boldsymbol{x}, \boldsymbol{y})$. Mimicking the treatment of Blake (1971) and Elasmi \& Feuillebois (2001), a stokeslet image with strength $-\boldsymbol{e}_{k}$ is introduced at the symmetric point of $\boldsymbol{y}$ with respect to $\Sigma_{u}$, that is, $\boldsymbol{y}^{\prime}=\boldsymbol{y}-2 y_{3} \boldsymbol{e}_{3}$. The Green's tensor is then searched as

$$
G_{j k}(\boldsymbol{x}, \boldsymbol{y})=G_{j k}^{\infty}(\boldsymbol{x}, \boldsymbol{y})-G_{j k}^{\infty}\left(\boldsymbol{x}, \boldsymbol{y}^{\prime}\right)+w_{j k}(\boldsymbol{x}, \boldsymbol{y}) .
$$

The unknown supplementary image $w_{j k}(\boldsymbol{x}, \boldsymbol{y})$ was derived by Khabthani (2004) with the use of a two-dimensional Fourier transform on $R_{1}^{\prime}, R_{2}^{\prime}$, with $\boldsymbol{R}^{\prime}=\boldsymbol{x}-\boldsymbol{y}^{\prime}$. Some details, as well as results for the pressure $H_{k}(\boldsymbol{x}, \boldsymbol{y})$ in $\mathscr{D}$ (recall (3.4)) and for the velocity and pressure in $\mathscr{S}$ and $\mathscr{D}^{\prime}$ (which will not be used in the present calculations), are provided in appendix B.

In giving the final result for the fluid velocity, it is useful to present the form of the inverse Fourier transform in the domain $R_{3}^{\prime}>0$ for a function $F(\xi)$ that is axisymmetric around the third coordinate $R_{3}^{\prime}$ as $\langle\xi F(\xi)\rangle$ with the transform

$$
\langle g\rangle\left(\rho, R_{3}^{\prime}\right)=\int_{0}^{\infty} \mathrm{J}_{0}(\rho \xi) e^{-R_{3}^{\prime} \xi} g(\xi) \mathrm{d} \xi,
$$

where $\rho=\sqrt{R_{1}^{\prime 2}+R_{2}^{\prime 2}}$ and $\mathrm{J}_{0}$ denotes the zeroth-order Bessel function. The result is then written as

$$
\begin{aligned}
w_{j k}(\boldsymbol{x}, \boldsymbol{y})= & {\left[\delta_{3 j}-x_{3} \frac{\partial}{\partial R_{j}^{\prime}}\right]\left\{\mathscr{J}_{k l} \frac{\partial\left\langle f_{1}\right\rangle}{\partial R_{l}^{\prime}}+\delta_{3 k}\left(\frac{\partial\left\langle f_{2}\right\rangle}{\partial R_{3}^{\prime}}+\left\langle f_{3}\right\rangle\right)\right\} } \\
& +\left[2 \sigma \sqrt{K} \frac{\partial}{\partial R_{3}^{\prime}}-1\right]\left\{\mathscr{J}_{k l} \frac{\partial^{2}\left\langle f_{4}\right\rangle}{\partial R_{j}^{\prime} \partial R_{l}^{\prime}}+\delta_{3 k}\left(\frac{\partial^{2}\left\langle f_{5}\right\rangle}{\partial R_{3}^{\prime} \partial R_{j}^{\prime}}+\frac{\partial\left\langle f_{6}\right\rangle}{\partial R_{j}^{\prime}}\right)\right\}
\end{aligned}
$$




$$
\begin{aligned}
& +\left\{\delta_{3 k} \delta_{j l} \sigma y_{3}+2 \sqrt{K}\left(\delta_{3 l} \mathscr{J}_{k j}-\delta_{3 j} \mathscr{J}_{k l}\right)\right. \\
& \left.+\left[2 K \sigma \delta_{3 k} \delta_{3 l}-y_{3} \sqrt{K}\left(\mathscr{J}_{k l}+\delta_{3 k} \delta_{3 l}\right)\right] \frac{\partial}{\partial R_{j}^{\prime}}\right\} \frac{\partial\left\langle f_{7}\right\rangle}{\partial R_{l}^{\prime}},
\end{aligned}
$$

with the symbol $\mathscr{J}_{k l}=\delta_{k 1} \delta_{1 l}+\delta_{k 2} \delta_{2 l}-\delta_{k 3} \delta_{3 l}$ and the transforms (3.14) of the functions $f_{q}(\xi)(q=1, \ldots, 7)$, which also depend on the slab thickness $e$, the permeability $K$ and the dimensionless coefficient $\sigma$. These functions are presented in appendix B (see (B 32), (B 33), (B 42) and (B 43)).

In the case $K=0$, the slab becomes impermeable and there is also no slip on it from (2.4) when furthermore $\sigma / \sqrt{K} \rightarrow \infty$. The no-slip condition $\boldsymbol{u}=0$ then applies on $\Sigma_{u}$. Recall that the Green's function was obtained for that case by Blake (1971). His result is recovered for $K \rightarrow 0$ and $\sigma / \sqrt{K} \rightarrow \infty$. Indeed, the definitions (B 42) and (B 43) show that $f_{1} \rightarrow-2 y_{3}$ and $f_{7} \rightarrow-2 / \sigma$ and the other functions vanish. The result (3.15) then becomes

$$
w_{j k}(\boldsymbol{x}, \boldsymbol{y})=-2 y_{3} \mathscr{J}_{k l}\left[\delta_{3 j}-x_{3} \frac{\partial}{\partial R_{j}^{\prime}}\right] \frac{\partial \phi}{\partial R_{l}^{\prime}}-2 \delta_{3 k} y_{3} \frac{\partial \phi}{\partial R_{j}^{\prime}},
$$

with the function (see Gradshteyn \& Ryzhik 1965)

$$
\phi=\int_{0}^{\infty} \mathrm{J}_{0}(\rho \xi) \mathrm{e}^{-R_{3}^{\prime} \xi} \mathrm{d} \xi=\frac{1}{R^{\prime}}, \quad R^{\prime}=\left|\boldsymbol{R}^{\prime}\right|=\left(\rho^{2}+R_{3}^{\prime 2}\right)^{1 / 2} .
$$

The limit (3.16) agrees with the result derived by Blake (1971). Henceforth, we discard the case $K=0$, which has been widely treated elsewhere.

\section{Numerical method for any particle to wall distance}

This section briefly presents the implemented numerical strategy to numerically invert the boundary integral equation (3.10).

Recall that the Green's tensor is given by (3.13) in which the stokeslet and its image are obtained from (3.3) and the supplementary image is given by (3.15) with the inverse transform (3.14) of the functions given in (B 42) and (B 43). The integral in the inverse transform (3.14) and its derivatives with respect to the components of $\boldsymbol{R}^{\prime}$ have to be calculated numerically. The formulae used in this calculation are given in appendix C.

The boundary integral equation (3.10) is discretized using, on the particle surface $S$, an $N$-node mesh constructed with $N_{e}$ isoparametric 6-node curved triangular boundary elements $\Delta_{e}$. The unknown density $\boldsymbol{d}$ on each isoparametric element $\Delta_{e}$ is thus expressed as a quadratic interpolation based on the values it takes at the nodes of $\Delta_{e}$. As a result, the discretized density is a $3 N$ unknown vector whose components are the values of the Cartesian components $d_{i}=\boldsymbol{d} \cdot \boldsymbol{e}_{i}$ at the nodal points. For any nodal point $\boldsymbol{x}$, the contribution of each element $\Delta_{e}$ to the integral on the left-hand side of (3.10) is then evaluated by distinguishing two alternative cases: either $\boldsymbol{x}$ is not or $\boldsymbol{x}$ is one of the nodal points of $\Delta_{e}$.

(a) If $\boldsymbol{x}$ is not one of the nodal points of $\Delta_{e}$, then each Cartesian component $G_{j k}(\boldsymbol{x}, \boldsymbol{y})$ is regular and the contribution of $\Delta_{e}$ is computed by using a standard Gaussian quadrature whose order, however, depends upon the location of $\boldsymbol{x}$ and is dictated by the so-called severity parameter proposed and numerically tested in Rezayat, Shippy \& Rizzo (1986). 
(b) If $\boldsymbol{x}$ is one of the nodal points of $\Delta_{e}$, then, practically, $\boldsymbol{x}$ may be part of either two or six $\Delta_{e}$ elements. Each component $G_{j k}(\boldsymbol{x}, \boldsymbol{y})$ exhibits on $\Delta_{e}$ a weakly singular behaviour originating from the $G_{j k}^{\infty}(\boldsymbol{x}, \boldsymbol{y})$ term in the (3.2) decomposition (recall that $R_{i j}(\boldsymbol{x}, \boldsymbol{y})$ is regular as $\left.\boldsymbol{x} \rightarrow \boldsymbol{y}\right)$. This singularity is adequately removed by using polar coordinates on $\Delta_{e}$ when treating the $G_{j k}^{\infty}(\boldsymbol{x}, \boldsymbol{y})$ term, whereas the regular contribution $R_{i j}(\boldsymbol{x}, \boldsymbol{y})$ is still treated as in $(a)$.

For further details regarding this key treatment of the integral in (3.10), the reader is directed to standard textbooks (see Brebbia, Telles \& Wrobel 1984; Bonnet 1995) and also to Sellier (2010). The discretized counterpart of (3.10) then becomes a linear system $\mathcal{A} \cdot \boldsymbol{X}=\mathcal{B}$, with the $3 N$-dimensional unknown discretized density vector $\boldsymbol{X}$ and a $3 N \times 3 N$ square, dense, non-symmetric so-called influence matrix $\mathcal{A}$. Such a system is finally solved by Gaussian elimination.

\section{Numerical results and discussion}

This section presents numerical results for a spherical or non-spherical particle (recall figure 1) with uniform density $\rho_{s}$, length scale $a$ and centre of volume $O^{\prime}$. We set $l=\boldsymbol{O O}^{\prime} \cdot \boldsymbol{e}_{3}$ and denote by $d$ the particle-slab gap. The porous slab has thickness $e$ and pores with size $\sqrt{K}$ smaller than $a$ and $d$. Finally, the slip length $b=\sqrt{K} / \sigma$ is smaller than $e$. For convenience, we introduce the following dimensionless permeability $K^{*}$, slab thickness $e^{*}$ and slab slip length $\lambda$ such that

$$
K^{*}=\frac{K}{a^{2}} \ll \min \left(1, \frac{d^{2}}{a^{2}}\right), \quad e^{*}=\frac{e}{a}, \quad d^{*}=\frac{d}{a}, \quad \lambda=\frac{\sqrt{K^{*}}}{\sigma}<e^{*} .
$$

\subsection{Case of a spherical particle}

\subsubsection{Normalized quantities and convergence}

The sphere radius is $a$ and thus $d^{*}=l / a-1$. Here we confine our attention to the calculation of the hydrodynamic force $\boldsymbol{F}_{h}$ and torque $\boldsymbol{\Gamma}_{h}$ (about $O^{\prime}$ ) exerted on the sphere when it moves at velocities $\boldsymbol{U}$ and $\boldsymbol{\Omega}$ with $\boldsymbol{g}=\boldsymbol{u}_{\infty}=0$ and also to the sphere motion when submitted to the gravity field $\boldsymbol{g}$ and/or to the external flow (2.13a) defined by $\left(\gamma_{1}, \gamma_{2}\right)$. For $d \gg a$ it is well known that

$$
\boldsymbol{F}_{h}=-6 \pi \mu a \boldsymbol{U}, \quad \boldsymbol{\Gamma}_{h}=-8 \pi \mu a^{3} \boldsymbol{\Omega},
$$

and that the sphere settles at the velocity $\boldsymbol{U}^{s}=2 a^{2}\left(\rho_{s}-\rho\right) \boldsymbol{g} /(9 \mu)$ in the gravity field $\boldsymbol{g}$. Moreover, the Faxen relationships (see Happel \& Brenner 1991) show that, when distant and submitted to the external flow $(2.13 a)$, the freely suspended sphere moves with the following translational and rotational velocities:

$$
\boldsymbol{U}=\left[\gamma_{1}\left(l+\frac{\sqrt{K}}{\sigma}\right)+\gamma_{2}\left(l^{2}+\frac{a^{2}}{3}-2 K\right)\right] \boldsymbol{e}_{1}, \quad \boldsymbol{\Omega}=\left[\frac{\gamma_{1}}{2}+\gamma_{2} l\right] \boldsymbol{e}_{2} .
$$

For a sphere located near the porous slab, symmetries then easily allow us to show that

$$
\begin{gathered}
\boldsymbol{F}_{h}=-6 \pi \mu a\left[\left(f_{11} U_{1}+a f_{12} \Omega_{2}\right) \boldsymbol{e}_{1}+f_{33} U_{3} \boldsymbol{e}_{3}\right], \\
\boldsymbol{\Gamma}_{h}=-8 \pi \mu a^{2}\left[c_{21} U_{1} \boldsymbol{e}_{2}+a c_{22} \Omega_{2} \boldsymbol{e}_{2}\right], \\
\boldsymbol{U}=\frac{2}{9} \frac{a^{2}}{\mu}\left(\rho_{S}-\rho\right)\left[u_{1}^{s} g_{1} \boldsymbol{e}_{1}+u_{3}^{s} g_{3} \boldsymbol{e}_{3}\right], \quad \boldsymbol{\Omega}=\frac{2}{9} \frac{a}{\mu}\left(\rho_{s}-\rho\right) \omega_{2}^{s} g_{1} \boldsymbol{e}_{2} \quad \text { if } \boldsymbol{u}_{\infty}=0,
\end{gathered}
$$




\begin{tabular}{|c|c|c|c|c|c|}
\hline$K^{*}$ & $d^{*}$ & $N$ & $f_{11}$ & $c_{21}$ & $f_{33}$ \\
\hline & $10 / 9-1$ & $\begin{array}{c}74 \\
242 \\
1058\end{array}$ & $\begin{array}{l}2.1649 \\
2.1750 \\
2.1732\end{array}$ & $\begin{array}{l}0.0767 \\
0.0810 \\
0.0789\end{array}$ & $\begin{array}{l}9.1501 \\
9.4666 \\
9.5035\end{array}$ \\
\hline & 1 & $\begin{array}{c}74 \\
242 \\
1058\end{array}$ & $\begin{array}{l}1.3755 \\
1.3796 \\
1.3800\end{array}$ & $\begin{array}{l}0.0046 \\
0.0050 \\
0.0049\end{array}$ & $\begin{array}{l}2.1049 \\
2.1131 \\
2.1141\end{array}$ \\
\hline & $10 / 9-1$ & $\begin{array}{c}74 \\
242 \\
1058\end{array}$ & $\begin{array}{l}1.8771 \\
1.8859 \\
1.8869\end{array}$ & $\begin{array}{l}0.0801 \\
0.0813 \\
0.0810\end{array}$ & $\begin{array}{l}4.4368 \\
4.4727 \\
4.4739\end{array}$ \\
\hline & 1 & $\begin{array}{c}74 \\
242 \\
1058\end{array}$ & $\begin{array}{l}1.3508 \\
1.3548 \\
1.3552\end{array}$ & $\begin{array}{l}0.0051 \\
0.0055 \\
0.0054\end{array}$ & $\begin{array}{l}1.9818 \\
1.9890 \\
1.9899\end{array}$ \\
\hline
\end{tabular}

TABLE 1. Computed friction coefficients $f_{11}, c_{21}$ and $f_{33}$ versus the number $N$ of nodal points on the sphere surface for $e^{*}=1, \sigma=1$, and for two values of the sphere-slab normalized gap $d^{*}=l / a-1$ and of the normalized permeability $K^{*}$.

$$
\begin{gathered}
\boldsymbol{U}=\left[\gamma_{1}\left(l+\frac{\sqrt{K}}{\sigma}\right) u_{1}^{l}+\gamma_{2}\left(l^{2}+\frac{a^{2}}{3}-2 K\right) u_{1}^{q}\right] \boldsymbol{e}_{1}, \\
\boldsymbol{\Omega}=\left(\frac{\gamma_{1}}{2} \omega_{2}^{l}+\gamma_{2} l \omega_{2}^{q}\right) \boldsymbol{e}_{2} \quad \text { if } \boldsymbol{g}=\mathbf{0},
\end{gathered}
$$

where $U_{i}, \Omega_{i}, g_{i}$ denote the $i$ component of $\boldsymbol{U}, \boldsymbol{\Omega}, \boldsymbol{g}$, respectively. The components $U_{2}, \Omega_{1}$ in (5.4), (5.5) and $g_{2}$ in (5.6) were not considered for simplicity, since results are analogous. The quantities $f_{11}, c_{21}, c_{22}, f_{12}, f_{33}$ in (5.4) and (5.5) are normalized friction coefficients, and the normalized velocities $u_{1}^{s}, \omega_{2}^{s}, u_{3}^{s}, u_{1}^{l}, \quad \omega_{2}^{l}, u_{1}^{q}, \omega_{2}^{q}$ in (5.6) and (5.7) may be interpreted as mobilities. These normalized quantities are here computed by using an $N$-node mesh on the sphere surface. For $K^{*}=0$ (case of a no-slip wall) it was checked that the present computations retrieve the results obtained in Sellier (2005), which are in full agreement with those of Hsu \& Ganatos (1994).

It was found that taking $N=242$ is sufficient to ascertain a relative accuracy of order $10^{-3}$ for all normalized quantities (except for $c_{12}$, for which the accuracy is of the order of $10^{-2}$ for small $K^{*}$ and small $d^{*}$ ) when $e^{*}$ is in the range $[0.5,10]$ and $K^{*}$ in the range $\left[10^{-4}, 10^{-2}\right]$. As illustrated in table 1 for $f_{11}, c_{21}, f_{33}$ in the case $e^{*}=1$, $N=242$ is sufficient to obtain a good accuracy both for a sphere located very close to the slab $\left(d^{*}=10 / 9-1\right)$ and at some distance from the slab $\left(d^{*}=1\right)$.

Our numerical results also show that each normalized quantity turns out to be nearly insensitive to the porous slab normalized thickness $e^{*}$ for given values of $\sigma$ and $K^{*}$. This behaviour is illustrated in table 2 for the coefficients $f_{11}, c_{21}$ and $f_{33}$. Table 2 also shows that, by contrast, these coefficients $f_{11}, c_{21}$ and $f_{33}$ are very dependent upon $\left(\sigma, K^{*}\right)$ and the sphere-slab normalized gap $d^{*}$. The normalized slip length $\lambda=\sqrt{K^{*}} / \sigma$ is indicated in the table. By comparing values of the friction coefficients obtained for the same $\lambda$ and different $K^{*}$, it is clear that both the slip and porosity of the porous slab play a significant role. 


\begin{tabular}{|c|c|c|c|c|c|c|c|c|c|c|}
\hline & \multirow[b]{2}{*}{$\sigma$} & \multirow[b]{2}{*}{$d^{*}=\frac{l}{a}-1$} & \multicolumn{4}{|c|}{$K^{*}=10^{-4}$} & \multicolumn{4}{|c|}{$K^{*}=10^{-2}$} \\
\hline & & & $\lambda$ & $e^{*}=0.5$ & $e^{*}=1$ & $e^{*}=10$ & $\lambda$ & $e^{*}=0.5$ & $e^{*}=1$ & $e^{*}=10$ \\
\hline \multirow{9}{*}{$f_{11}$} & & $10 / 9-1$ & & 1.9351 & 1.9353 & 1.9354 & & $*$ & $*$ & 1.3309 \\
\hline & 0.1 & 1.0000 & 0.1 & 1.3581 & 1.3581 & 1.3581 & 1 & * & * & 1.2328 \\
\hline & & 9.0000 & & 1.0586 & 1.0586 & 1.0586 & & * & * & 1.0538 \\
\hline & & $10 / 9-1$ & & 2.1747 & 2.1750 & 2.1750 & & 1.8771 & 1.8859 & 1.8887 \\
\hline & 1 & 1.0000 & 0.01 & 1.3796 & 1.3796 & 1.3796 & 0.1 & 1.3531 & 1.3548 & 1.3556 \\
\hline & & 9.0000 & & 1.0591 & 1.0591 & 1.0591 & & 1.0585 & 1.0585 & 1.0586 \\
\hline & & $10 / 9-1$ & & 2.2103 & 2.2106 & 2.2107 & & 2.0817 & 2.0924 & 2.0956 \\
\hline & 10 & 1.0000 & 0.001 & 1.3819 & 1.382 & 1.382 & 0.01 & 1.3738 & 1.3756 & 1.3764 \\
\hline & & 9.0000 & & 1.0592 & 1.0592 & 1.0592 & & 1.059 & 1.0591 & 1.0591 \\
\hline \multirow{9}{*}{$c_{21}$} & & $10 / 9-1$ & & 0.0321 & 0.0319 & 0.0319 & & $*$ & $*$ & -0.0621 \\
\hline & 0.1 & 1.0000 & 0.1 & 0.0037 & 0.0037 & 0.0037 & 1 & $*$ & $*$ & -0.0094 \\
\hline & & 9.0000 & & 0.0000 & 0.0000 & 0.0000 & & $*$ & $*$ & -0.0000 \\
\hline & & $10 / 9-1$ & & 0.0812 & 0.0810 & 0.0809 & & 0.0864 & 0.0813 & 0.0798 \\
\hline & 1 & 1.0000 & 0.01 & 0.0050 & 0.0050 & 0.0050 & 0.1 & 0.0061 & 0.0055 & 0.0051 \\
\hline & & 9.0000 & & 0.0000 & 0.0000 & 0.0000 & & 0.0000 & 0.0000 & 0.0000 \\
\hline & & $10 / 9-1$ & & 0.0870 & 0.0868 & 0.0867 & & 0.1664 & 0.1608 & 0.1591 \\
\hline & 10 & 1.0000 & 0.001 & 0.0051 & 0.0050 & 0.0050 & 0.01 & 0.0079 & 0.0072 & 0.0069 \\
\hline & & 9.0000 & & 0.0000 & 0.0000 & 0.0000 & & 0.0000 & 0.0000 & 0.0000 \\
\hline \multirow{9}{*}{$f_{33}$} & & $10 / 9-1$ & & 7.1748 & 7.1912 & 7.1963 & & $*$ & $*$ & 3.8288 \\
\hline & 0.1 & 1.0000 & 0.1 & 2.0364 & 2.0367 & 2.0369 & 1 & * & * & 1.7802 \\
\hline & & 9.0000 & & 1.1245 & 1.1245 & 1.1245 & & $*$ & * & 1.1156 \\
\hline & & $10 / 9-1$ & & 9.4325 & 9.4666 & 9.4764 & & 4.1366 & 4.4727 & 4.6263 \\
\hline & 1 & 1.0000 & 0.01 & 2.1128 & 2.1131 & 2.1133 & 0.1 & 1.9647 & 1.9890 & 2.0031 \\
\hline & & 9.0000 & & 1.1257 & 1.1257 & 1.1257 & & 1.1237 & 1.1241 & 1.1244 \\
\hline & & $10 / 9-1$ & & 9.9676 & 10.007 & 10.018 & & 4.4019 & 4.8057 & 4.9916 \\
\hline & 10 & 1.0000 & 0.001 & 2.1223 & 2.1227 & 2.1229 & 0.01 & 2.0247 & 2.0529 & 2.0689 \\
\hline & & 9.0000 & & 1.1258 & 1.1258 & 1.1259 & & 1.1248 & 1.1253 & 1.1256 \\
\hline
\end{tabular}

TABLE 2. Sensitivity, for different sphere locations $d^{*}=l / a-1$, of the friction coefficients $f_{11}, c_{21}$ and $f_{33}$ to the parameters $\sigma, e^{*}$ and $K^{*}$ when using 242 nodal points on the sphere boundary. Stars indicate values of $\left(K^{*}, \sigma\right)$ such that $\lambda \geqslant e^{*}$, i.e. values not complying with conditions (5.1). The normalized slip length $\lambda=\sqrt{K^{*}} / \sigma$ is also indicated in the table. 


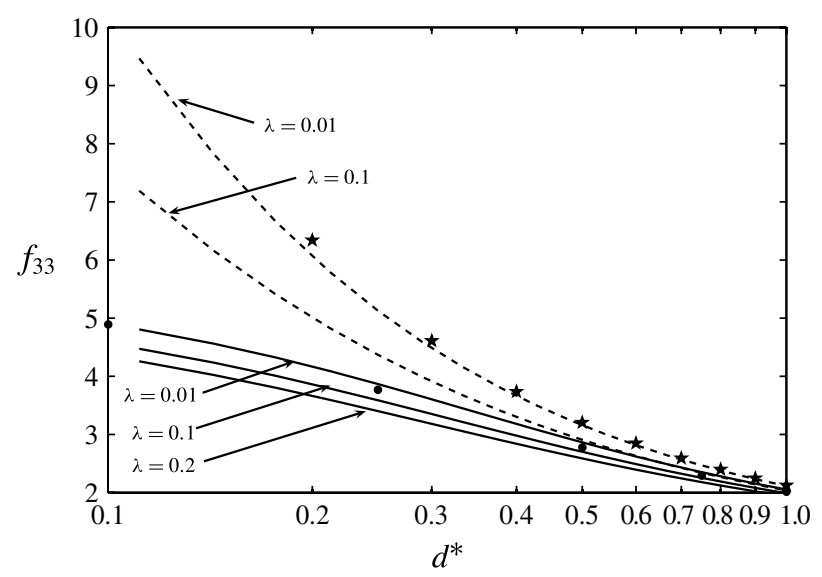

FIGURE 3. Friction coefficient $f_{33}$ versus $d^{*} \geqslant 10 / 9-1$ for a weakly permeable slab with $K^{*}=10^{-4}$ (dashed curves) and a slightly permeable slab with $K^{*}=10^{-2}$ (solid curves). For comparison, results obtained by Michalopoulou et al. (1992) for $K^{*}=10^{-2}$ and $\lambda=0.1$ are indicated with $\bullet$ symbols and those of Brenner (1961) and Maude (1961) by $\star$ symbols. The porous slab thickness is $e^{*}=1$.

The largest variation with $e^{*}$ occurs for $f_{33}$ at $K^{*}=10^{-2}$ and at short gaps $d^{*}$, which is the most stringent configuration of lubrication. Note that at $K^{*}=10^{-2}$, when $e^{*}$ varies from 1 to 0.5 , then $e^{*} / \sqrt{K^{*}}=e / \sqrt{K}$ varies from 10 to 5 , which physically means that the number of pores across the slab is reduced from about 10 to 5 . Obviously, Darcy's model would be inappropriate if there were only a few pores across the slab. As announced in the introduction, Brinkman's model would be a better model to describe the flow behaviour in such a medium.

We thus restrict our numerical investigation to the domain $e^{*} / \sqrt{K^{*}} \geqslant 10$. In this domain, the low variation with the slab thickness makes it possible to further restrict our investigation to the case $e^{*}=1$.

\subsubsection{Friction coefficients}

The coefficient $f_{33}$ for a sphere translating normal to the permeable porous slab with thickness $e^{*}=1$, normalized permeability $K^{*}$ and slip length $\lambda$ is plotted in figure 3 .

As for a solid boundary, $f_{33}$ monotonically decreases as $d^{*}$ increases (i.e. as the sphere moves away from the slab) for any prescribed setting $\left(K^{*}, \lambda\right)$. Not surprisingly (recall (2.2) and (2.3b)), for a weakly permeable slab $\left(K^{*}=10^{-4}\right)$ and $\lambda=0.01$, the value of $f_{33}$ is close to the one predicted for a solid impermeable and no-slip plane wall by Brenner (1961) and Maude (1961) using the bipolar coordinates method. Still for $K^{*}=10^{-4}$, note that $f_{33}$ is quite sensitive (especially for a sphere close to the slab) to the slip length $\lambda$. For a given triplet $\left(K^{*}, \lambda, d^{*}\right)$, the coefficient $f_{33}$ drops as $K^{*}$ increases (as illustrated by the curves associated with $\left(K^{*}, \lambda\right)=\left(10^{-4}, 10^{-2}\right)$ and $\left.\left(K^{*}, \lambda\right)=\left(10^{-2}, 10^{-2}\right)\right)$ because more fluid is allowed to flow across the porous slab as $K^{*}$ increases. Note that, as expected, for $K^{*}=10^{-2}$ the coefficient $f_{33}$ decreases as the slip length $\lambda$ increases, since the shear rate of the flow taking place in the slab-sphere gap is smaller when $\lambda$ is bigger and thus the lubrication force is then smaller. Using the bipolar coordinates method, Michalopoulou et al. (1992) considered the axisymmetric translation of a solid sphere towards another motionless and porous 

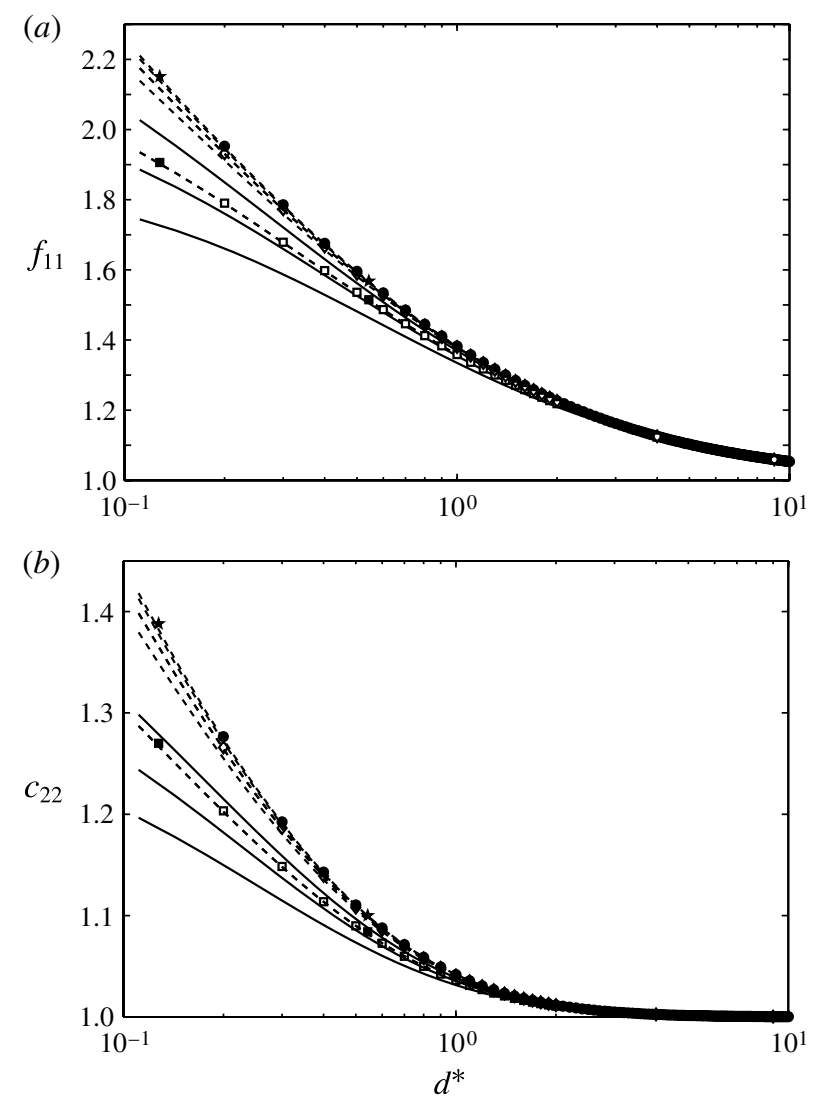

FIGURE 4. (a) Force friction coefficient $f_{11}$ and $(b)$ torque friction coefficient $c_{22}$ versus $d^{*}$ for $K^{*}=10^{-4}$ (dashed lines; for $\lambda=0.1,0.02,0.01,0.01 / 3,0.001$ ) or $K^{*}=10^{-2}$ (solid lines; for $\lambda=0.2,0.1,0.1 / 3$ ). Both $f_{11}$ and $c_{22}$ increase when $\lambda$ decreases (use this property to retrieve the value of $\lambda$ associated with each curve). Also plotted for comparison are the results obtained for a slipping and impermeable boundary by Davis, Kezirian \& Brenner (1994) using the bipolar coordinates method ( $\square$ for $\lambda=0.1$, and $\diamond$ for $\lambda=0.01$ ) and by Luo $\&$ Pozrikidis (2008) using the boundary integral equations technique ( $\boldsymbol{\square}$ for $\lambda=0.1$ ), and the results obtained for a solid wall and a no-slip particle by Luo \& Pozrikidis (2008) ( $\star$ for $\lambda=0)$ and by Chaoui \& Feuillebois (2003) $(\bullet)$.

sphere, the translational velocity being aligned with the line of centres. We thus compare in figure 3 our results against the predictions of Michalopoulou et al. (1992) for a small solid sphere approaching a very large porous sphere. Since our results are weakly sensitive to the slab thickness $e^{*}$ (see previous remarks), good agreement is obtained.

The force and torque exerted on a sphere translating or rotating parallel with the slab are characterized by the coefficients $\left(f_{11}, c_{21}\right)$ or $\left(f_{12}, c_{22}\right)$, respectively (recall (5.4) and (5.5)). The coefficients $f_{11}$ and $c_{22}$, plotted in figure 4 , have comparable magnitudes. Note that, as for a solid boundary, $f_{11}$ is weaker than $f_{33}$ for any prescribed value of $\left(d^{*}, K^{*}, \lambda\right)$. This means that it is easier to move (translate or rotate) the sphere parallel with the slab than normal to it. For the weak permeability $K^{*}=10^{-4}$, the porous slab behaves as an impermeable boundary of solid nature if $\lambda \lesssim 0.01$ 

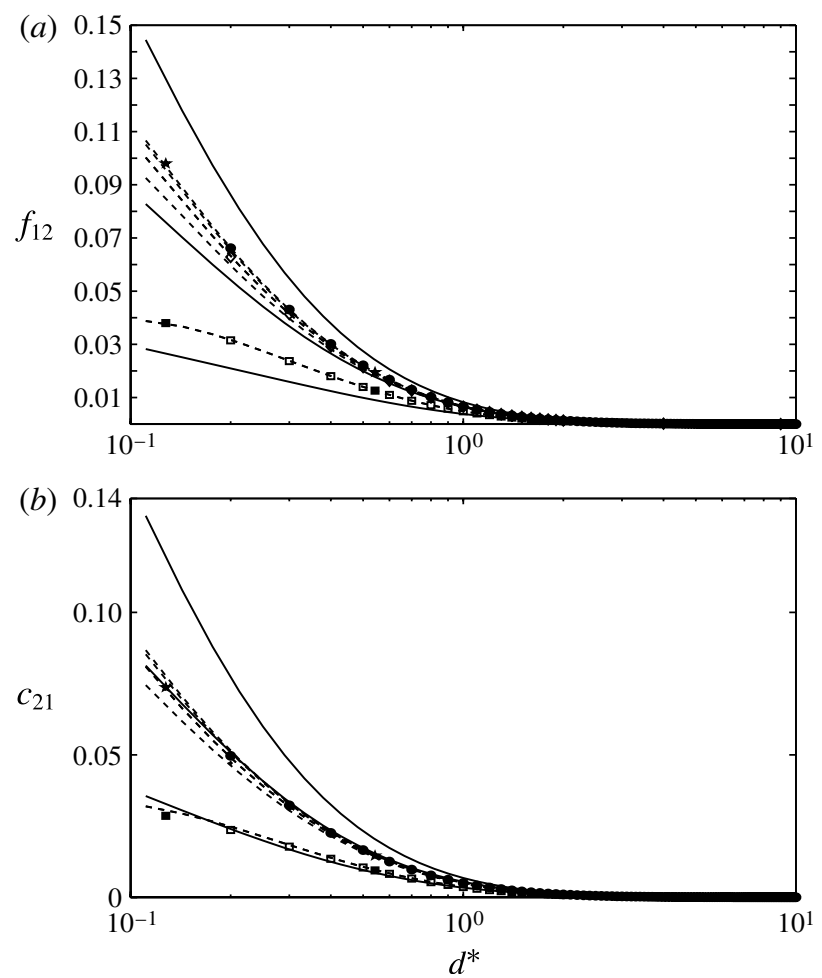

FIgURE 5. (a) Force friction coefficient $f_{12}$ and $(b)$ torque friction coefficient $c_{21}$. As in figure 4, dashed or solid curves are employed for $K^{*}=10^{-4}$ or $K^{*}=10^{-2}$, respectively, and the curves hierarchy versus $\lambda$ is the same as in figure 4 . In addition, comparisons with Davis et al. (1994) ( $\square$ for $\lambda=0.1$, and $\diamond$ for $\lambda=0.01$ ), Luo \& Pozrikidis (2008) ( $\square$ for $\lambda=0.1$, and $\star$ for $\lambda=0$ ) and Chaoui \& Feuillebois (2003) (•) are given.

(see the comparisons with Chaoui \& Feuillebois (2003) and Luo \& Pozrikidis (2008) for $\lambda=0$ ) or of slipping nature for $\lambda$ large enough (see the comparisons with Davis et al. (1994) and Luo \& Pozrikidis (2008) for $\lambda=0.1$ ). The coefficients $f_{12}$ and $c_{21}$, displayed in figure 5 , exhibit the same behaviours versus $\left(d^{*}, K^{*}, \lambda\right)$ as the coefficients $f_{11}$ and $c_{22}$ but are of weaker magnitudes. Note that, under our definitions (5.4), (5.5) and (2.10), it turns out that here $B_{12}=6 \pi a^{2} f_{12}$ and $C_{21}=8 \pi a^{2} c_{21}$. As noted at the end of $\S 2.1$, as $d^{*}$ becomes large whatever $K^{*}$, or as $K^{*} \rightarrow 0$ whatever $d^{*}$, one expects (using the reciprocity identity) the difference $3 f_{12}-4 c_{21}$ to vanish. However, in general $3 f_{12} \neq 4 c_{21}$. These predictions fully agree with the behaviour of the computed quantity $4 c_{21} / 3 f_{12}-1$ plotted in figure 6 for $K^{*}=10^{-4}$ and $K^{*}=10^{-2}$. As revealed by figure $6(b)$ for $K^{*}=10^{-2}$, the difference $3 f_{12}-4 c_{21}$ is not negligible at least in the range $d^{*} \leqslant 1$.

\subsubsection{Settling velocities}

Here we present results for a settling sphere when $e^{*}=1$ and $K^{*}=10^{-2}$. The normalized translational velocities $u_{1}^{s}$ and $u_{3}^{s}$ (note that, under our definitions (5.4) and (5.6), it turns out that $u_{3}^{s}=1 / f_{33}$ ) and angular velocity $\omega_{2}^{s}$ are plotted in figure 7. Both quantities $u_{1}^{s}$ and $u_{3}^{s}$ increase with the slip length and decrease as the sphere approaches the slab. Not surprisingly, the sphere translates faster parallel with the slab 

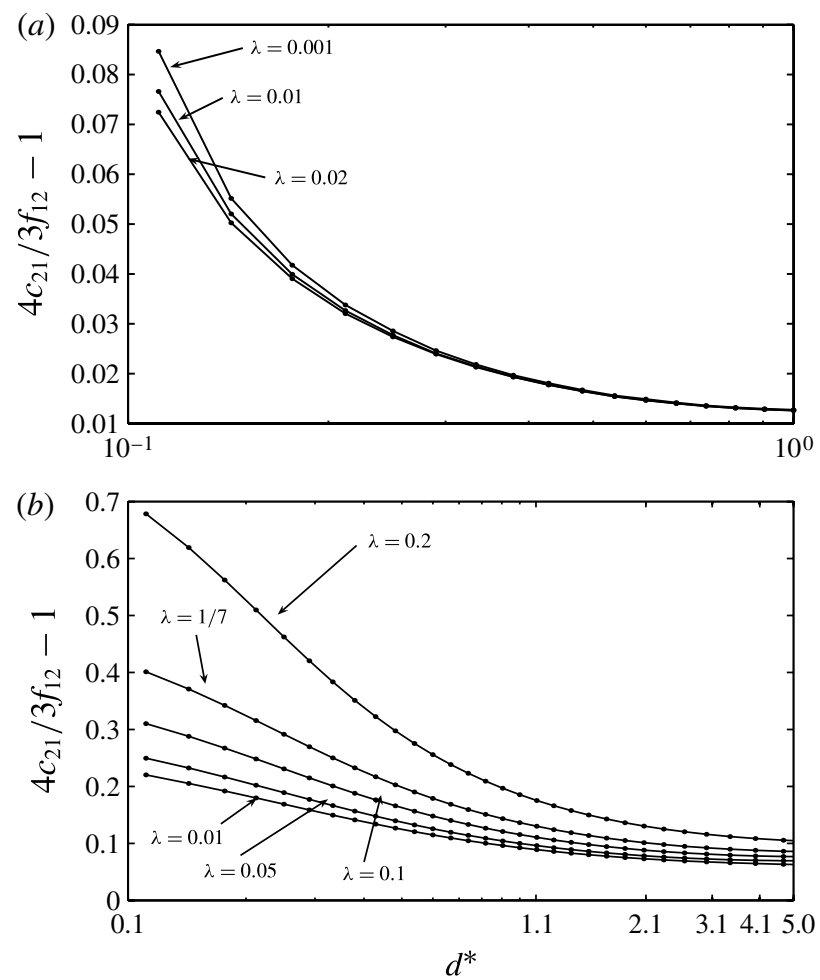

FIGURE 6. Quantity $4 c_{21} / 3 f_{12}-1$ versus the normalized particle-slab gap $d^{*}$ for $(a)$ the weak permeability $K^{*}=10^{-4}$ and $(b)$ the permeability $K^{*}=10^{-2}$.

than normal to it $\left(u_{1}^{s}>u_{3}^{s}\right)$. By contrast, the angular velocity $\omega_{2}^{s}$ is seen to decrease as $\lambda$ increases for any sphere location and to increase as the sphere approaches the slab. For $\lambda \geqslant 1 / 3$ the sphere experiences a very weak rotation because the slab then permits the fluid to flow parallel with $\boldsymbol{e}_{1}$ at a very low friction cost.

\subsubsection{External shear flows}

When freely suspended in either a linear or a quadratic ambient shear flow, the sphere migrates at translational and angular velocities with normalized components as defined by (5.7), $\left(u_{1}^{l}, \omega_{2}^{l}\right)$ and $\left(u_{1}^{q}, \omega_{2}^{q}\right)$, respectively. These velocities are plotted in figures 8 and 9. As the sphere approaches the slab, both $u_{1}^{l}$ and $\omega_{2}^{l}$ decrease. For a given sphere location $d^{*}$, these velocities increase with $K^{*}$ for prescribed $\lambda$; whereas, for a given permeability $K^{*}$, note that, as $\lambda$ increases, then $u_{1}^{l}$ increases, but $\omega_{2}^{l}$ may either increase (for $K^{*}=10^{-4}$ ) or decrease (for $K^{*}=10^{-2}$ ). For a low permeability, the results versus $\lambda$ are in good agreement with those for an impermeable slip wall obtained by bispherical coordinates in Loussaief (2008) and by BIM in Luo \& Pozrikidis (2008). For the quadratic shear flow, $u_{1}^{q}$ and $\omega_{2}^{q}$ exhibit the same behaviour as $u_{1}^{l}$ and $\omega_{2}^{l}$, except that, for large enough $d^{*}, \omega_{2}^{q}$ admits a minimum value when $\lambda$ is small enough and $K^{*}$ large (see the curve for $K^{*}=10^{-2}$ and $\lambda=0.1 / 3$ ).

\subsection{Case of a non-spherical particle}

For a sphere, the considered problems involved, by symmetries, 12 relevant normalized quantities defined by (5.4)-(5.7). Now, the non-spherical particles 

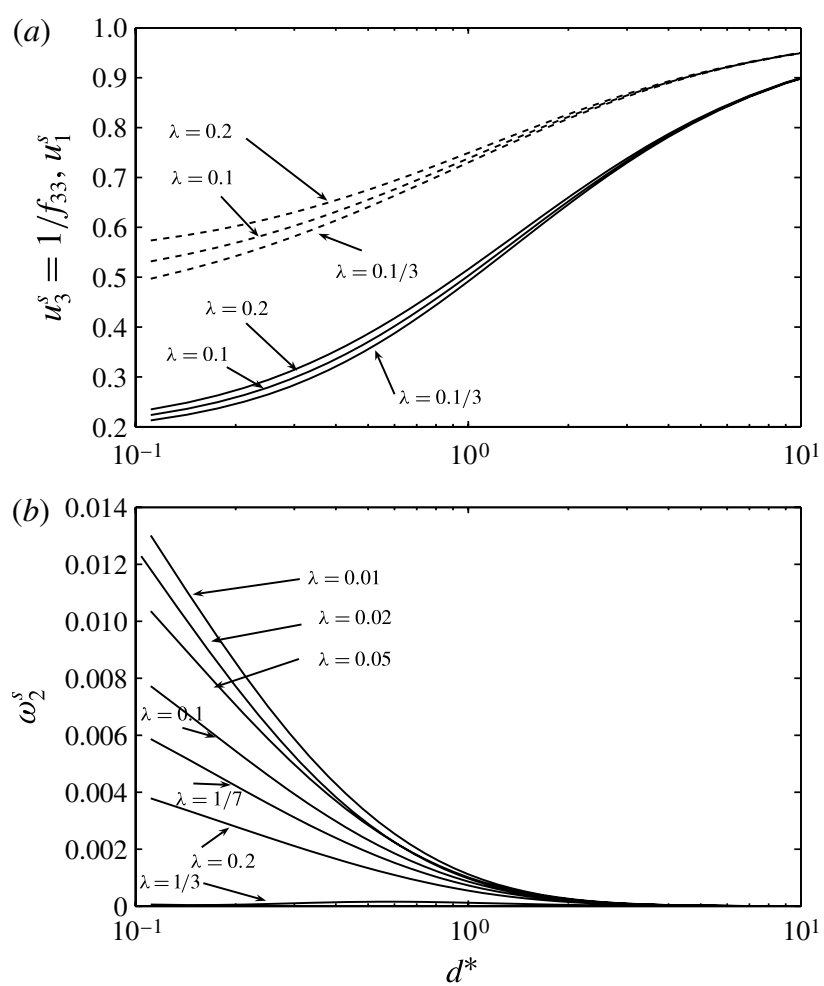

FIGURE 7. (a) Settling translational mobilities $u_{3}^{s}=1 / f_{33}$ and $u_{1}^{s}$ and $(b)$ angular mobility $\omega_{2}^{s}$ for $e^{*}=1$ and $K^{*}=10^{-2}$ versus $d^{*}$. In panel $(a)$ the solid and dashed lines represent $u_{3}^{s}$ and $u_{1}^{s}$, respectively.

often encountered in applications require a much larger number of parameters because of their shape and orientation. As previously mentioned, the proposed approach makes it possible to compute these quantities for arbitrarily shaped particles.

This key ability is illustrated here by dealing with an ellipsoidal particle like the one sketched in figure 10. The ellipsoid is described by its centre $O^{\prime}$, semi-axes $a_{1}, a_{2}, a_{3}$ and orientation defined by the angle $\alpha$. In the example cases presented below, we consider $a_{1}=1.2 a, a_{2}=a^{2} / a_{1}, a_{3}=a$ and $\alpha=\pi / 4$. For conciseness, we restrict our attention to rigid-body motions $(\boldsymbol{U}, \boldsymbol{\Omega})$ such that $\boldsymbol{U} \cdot \boldsymbol{e}_{2}=0$ and $\boldsymbol{\Omega} \wedge \boldsymbol{e}_{2}=\mathbf{0}$. In such circumstances, and adopting the scaling employed in (5.4)-(5.7) for a sphere with the same volume (i.e with radius $a$ ), we introduce normalized quantities such that

$$
\begin{gathered}
\boldsymbol{F}_{h}=-6 \pi \mu a\left\{\left[f_{11} U_{1}+f_{13} U_{3}\right] \boldsymbol{e}_{1}+a f_{12} \Omega_{2} \boldsymbol{e}_{1}+\left[f_{33} U_{3}+f_{31} U_{1}\right] \boldsymbol{e}_{3}+a f_{32} \Omega_{2} \boldsymbol{e}_{3}\right\}, \\
\boldsymbol{\Gamma}_{h}=-8 \pi \mu a^{2}\left\{\left[c_{21} U_{1}+c_{23} U_{3}\right] \boldsymbol{e}_{2}+a c_{22} \Omega_{2} \boldsymbol{e}_{2}\right\} \\
\boldsymbol{U}=\frac{2}{9} \frac{a^{2}}{\mu}\left(\rho_{S}-\rho\right)\left\{\left[u_{11}^{s} \boldsymbol{e}_{1}+u_{31}^{s} \boldsymbol{e}_{3}\right]\left(\boldsymbol{g} \cdot \boldsymbol{e}_{1}\right)+\left[u_{13}^{s} \boldsymbol{e}_{1}+u_{33}^{s} \boldsymbol{e}_{3}\right]\left(\boldsymbol{g} \cdot \boldsymbol{e}_{3}\right)\right\} \quad \text { if } \boldsymbol{u}_{\infty}=0, \\
\boldsymbol{\Omega}=\frac{2}{9} \frac{a}{\mu}\left(\rho_{s}-\rho\right)\left[\omega_{21}^{s}\left(\boldsymbol{g} \cdot \boldsymbol{e}_{1}\right)+\omega_{23}^{s}\left(\boldsymbol{g} \cdot \boldsymbol{e}_{3}\right)\right] \boldsymbol{e}_{2} \quad \text { if } \boldsymbol{u}_{\infty}=0,
\end{gathered}
$$



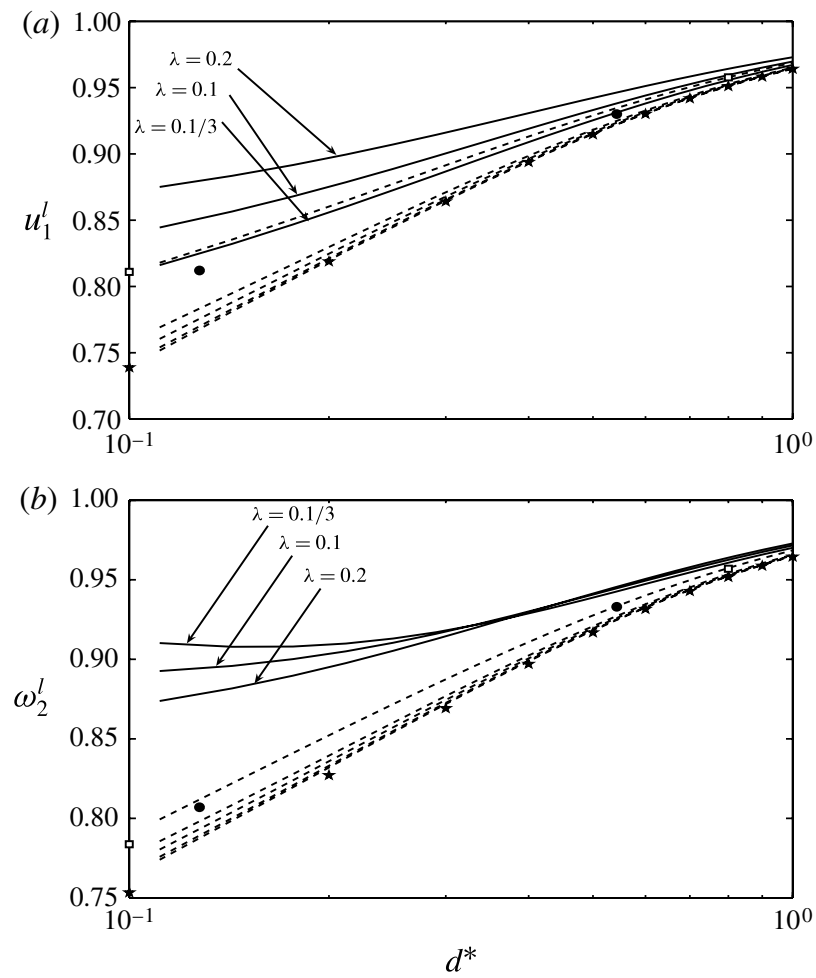

FIGURE 8. Mobilities $(a) u_{1}^{l}$ and $(b) \omega_{2}^{l}$ versus $d^{*}$. The solid and dashed lines represent $K^{*}=10^{-2}$ and $K^{*}=10^{-4}$, respectively. Values of the slip length $\lambda$ for $K^{*}=10^{-2}$ are shown. Values of $\lambda$ for $K^{*}=10^{-4}$ are $\lambda=0.01 / 3,0.01,0.02,0.1$ (from bottom to top). The results of Chaoui \& Feuillebois (2003) $(\star)$ for a solid impermeable no-slip wall and those of Loussaief (2008) ( $\square$ ) and Luo \& Pozrikidis (2008) (•) for an impermeable slipping wall with slip length $\lambda=0.1$ are shown for comparison.

$$
\begin{gathered}
\boldsymbol{U}=\left[\gamma_{1}\left(l+\frac{\sqrt{K}}{\sigma}\right)\right]\left[u_{1}^{l} \boldsymbol{e}_{1}+u_{3}^{l} \boldsymbol{e}_{3}\right] \\
+\gamma_{2}\left(l^{2}+\frac{a^{2}}{3}-2 K\right)\left[u_{1}^{q} \boldsymbol{e}_{1}+u_{3}^{q} \boldsymbol{e}_{3}\right] \quad \text { if } \boldsymbol{g}=\mathbf{0}, \\
\boldsymbol{\Omega}=\left(\frac{\gamma_{1}}{2} \omega_{2}^{l}+\gamma_{2} l \omega_{2}^{q}\right) \boldsymbol{e}_{2} \quad \text { if } \boldsymbol{g}=\mathbf{0} .
\end{gathered}
$$

Hence, we arrive at nine additional quantities: $f_{31}, f_{13}, f_{32}, c_{23}, u_{31}^{s}, u_{13}^{s}, \omega_{23}^{s}, u_{3}^{l}$ and $u_{3}^{q}$ (note that $\omega_{2}^{s}$ introduced in (5.6) for a sphere is renamed here $\omega_{21}^{s}$ for consistency of notation with the new term $\omega_{23}^{s}$ ). These new quantities vanish for a sphere. For arbitrarily shaped particles, they obey the relationships $f_{13}=f_{31}$ and $4 c_{23}=3 f_{32}$ when $K^{*}=0$ or for a distant porous slab; however, in general, $f_{13} \neq f_{31}, 4 c_{23} \neq 3 f_{32}$ and $4 c_{21} \neq 3 f_{12}$. For the selected ellipsoid, all the coefficients arising in (5.8)-(5.13) depend upon $K^{*}, e^{*}$ and $d^{*}=l / a-1$, with $l=\boldsymbol{O O}^{\prime} \cdot \boldsymbol{e}_{3}$. Like in the case of a sphere, it was found that taking $N=242$ nodal points on the boundary is sufficient to provide a good precision on the dimensionless coefficients. This is illustrated in table 3 for the example case $e^{*}=1, K^{*}=10^{-2}$ and $\lambda=1 / 3$. 

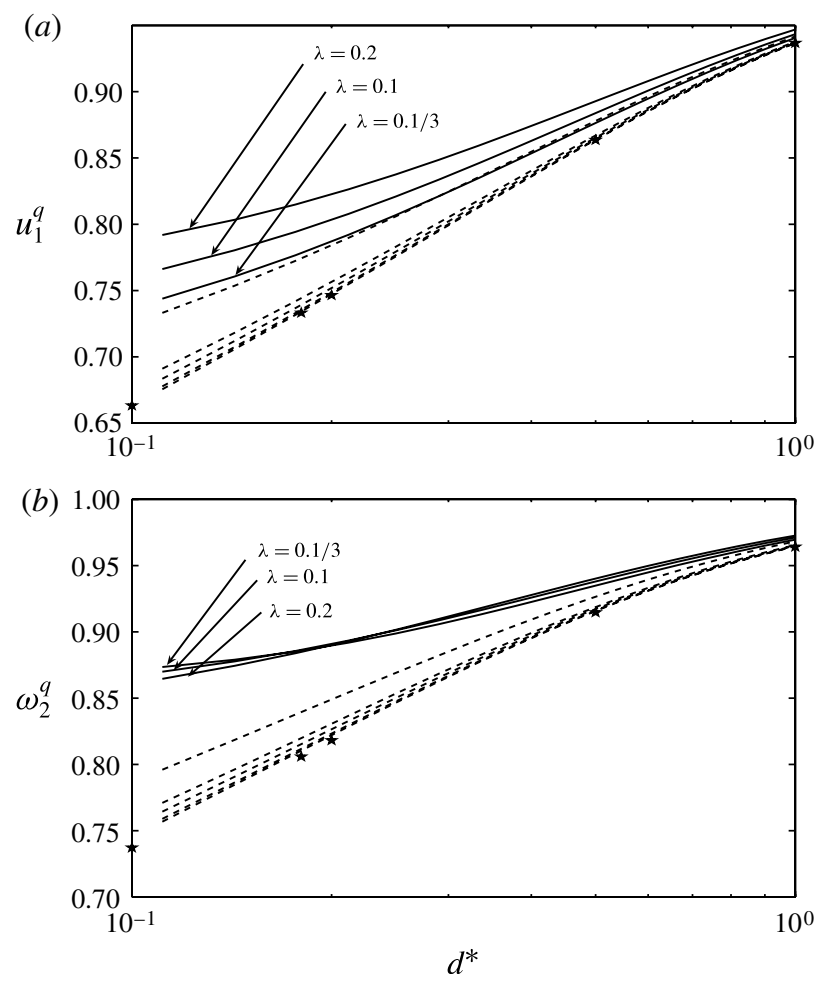

FIGURE 9. Mobilities $(a) u_{1}^{q}$ and $(b) \omega_{2}^{q}$ versus $d^{*}$, with solid or dashed lines for $K^{*}=10^{-2}$ or $K^{*}=10^{-4}$, respectively. For $K^{*}=10^{-4}$, values of the slip length are $\lambda=0.01 / 3,0.01,0.02$ and 0.1 (from bottom to top). Analytical results obtained by Pasol, Sellier \& Feuillebois (2006) $(\star)$ for an impermeable no-slip wall are compared with our results when the slip length $\lambda=0.01 / 3$ and $K^{*}=10^{-4}$.

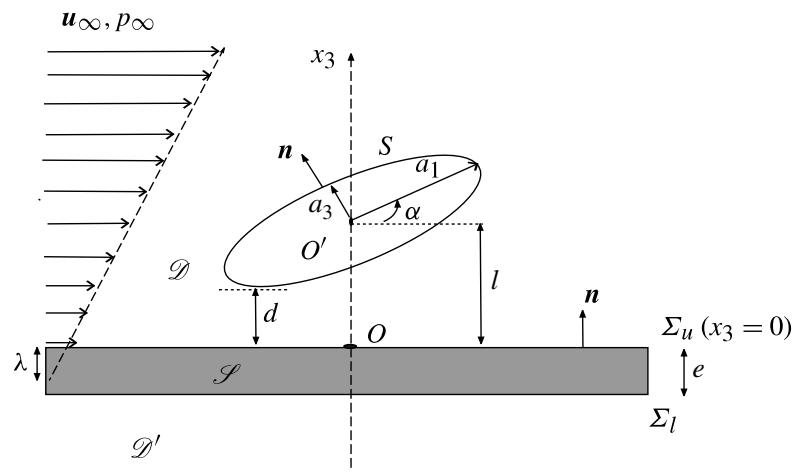

FIGURE 10. A solid ellipsoidal particle with centre $O^{\prime}$ and semi-axes $a_{1}, a_{2}$ (normal to the plane of the figure) and $a_{3}$ is freely suspended in a linear ambient shear flow with velocity $\boldsymbol{u}_{\infty}$ along a porous slab $\mathscr{S}$. Its orientation with respect to $\Sigma_{u}$ is given by the angle $\alpha$.

As observed in figure 11, the coefficients $f_{11}, f_{33}, c_{21}$ and $f_{12}$ exhibit the same trends as for a spherical particle (see figures $3-5$ ). The new friction coefficients $f_{31}, f_{13}, c_{23}$ 

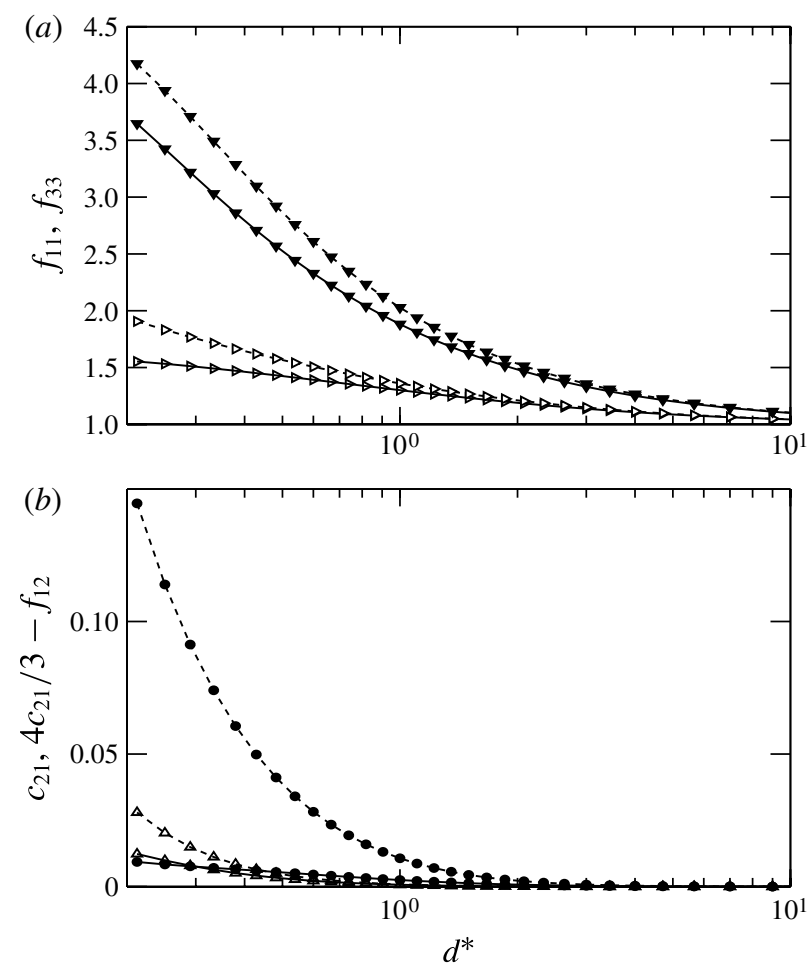

FIGURE 11. Normalized friction coefficients $f_{11}, f_{33}, c_{21}$ and $f_{12}$ versus $d^{*}$ for $\lambda=1 / 30$ (dashed lines), $\lambda=1 / 3$ (solid lines) and $\left(e^{*}, K^{*}\right)=\left(1,10^{-2}\right):(a) f_{11}(\triangleright)$ and $f_{33}(\boldsymbol{\nabla}) ;(b) c_{21}$ $(\bullet)$ and $4 c_{21} / 3-f_{12}(\triangle)$.

and $f_{32}$, plotted in figure 12, increase as the ellipsoid approaches the porous slab and decrease as $\lambda$ increases for a given ellipsoid location. In the gravity field $\boldsymbol{g}=g \boldsymbol{e}_{1}$, the ellipsoid rotates parallel with $\boldsymbol{e}_{2}$ and mainly translates in the direction of the applied gravity. Indeed, the mobility $u_{13}^{s}$ is small and slowly increases as the ellipsoid approaches the slab (as depicted in figure 13a). Note that, whereas $u_{11}^{s}$ increases with $\lambda$, the opposite behaviour is observed for $u_{31}^{s}$. A similar remark holds for $u_{33}^{s}$ and $u_{13}^{s}$. The angular mobility $\omega_{21}^{s}$ (for $\boldsymbol{g} \wedge \boldsymbol{e}_{1}=\mathbf{0}$ ) decreases as $\lambda$ increases and becomes very weak for $\lambda \gtrsim 1 / 3$, whereas the mobility $\omega_{23}^{s}$ (for $\boldsymbol{g} \wedge \boldsymbol{e}_{3}=\mathbf{0}$ ) depends weakly upon $\lambda$. This difference is linked to the flow field $\boldsymbol{u}$ near the porous slab with two cases:

(i) when $\boldsymbol{g}$ is parallel with the slab, the tangential velocity on the slab is not negligible and is 'triggered' by the slip length $\lambda$;

(ii) when $\boldsymbol{g}$ is normal to the slab, the tangential velocity is weak and the flow near the slab is nearly insensitive to $\lambda$ and is driven by the permeability $K^{*}$ (which clearly controls the velocity component $\boldsymbol{u} \cdot \boldsymbol{e}_{3}$ ).

Finally, we plot in figure 14 the mobilities $u_{1}^{q}, u_{3}^{q}$ and $\omega_{2}^{q}$. It turns out that $u_{3}^{q}$ is small when compared with $u_{1}^{q}$. In addition, the mobility $u_{3}^{q}$ increases as the ellipsoid approaches the slab and also decreases as $\lambda$ increases (in contrast to the mobility $u_{1}^{q}$ ). 

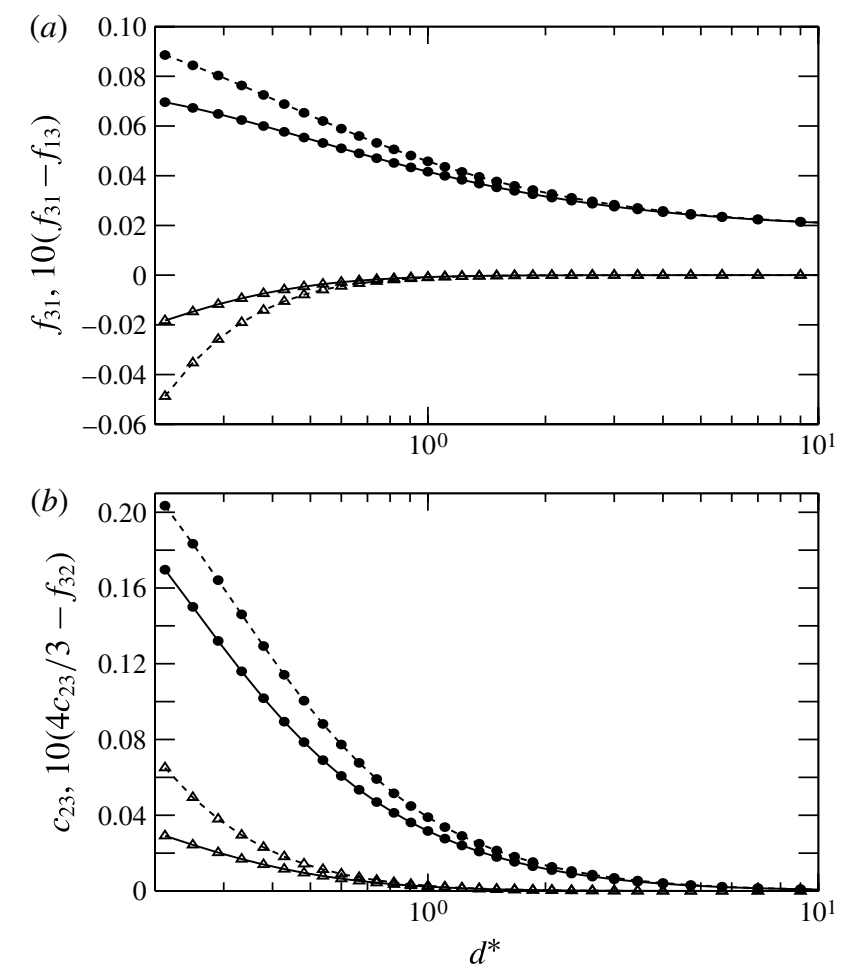

FIGURE 12. Normalized friction coefficients $f_{31}, f_{13}, c_{23}$ and $f_{32}$ versus $d^{*}$ for $\lambda=1 / 30$ (dashed lines), $\lambda=1 / 3$ (solid lines) and $\left(e^{*}, K^{*}\right)=\left(1,10^{-2}\right):(a) f_{31}(\bullet)$ and $10\left(f_{31}-f_{13}\right)(\triangle)$; (b) $c_{23}(\bullet)$ and $10\left(4 c_{23} / 3-f_{32}\right)(\triangle)$.

\section{Conclusion}

The coupled Stokes-Darcy problem with Beavers and Joseph slip boundary condition on the slab interfaces is solved here by a novel 'indirect boundary integral method' (IBIM), using the appropriate Green's tensor. The expression of this Green's tensor in the fluid domain is displayed here (complementary terms that are not directly used in the IBIM are also provided in appendix B). The unknown is a density $\boldsymbol{d}$ on the particle surface, defined up to $p_{0} \boldsymbol{n}$, where $p_{0}$ is a constant. This density is not the stress on the surface, yet provides the force and torque on the particle by simple quadratures. This approach appears to be simpler than the classical BIM, which would involve complicated terms on the porous slab surface.

The resulting integral equation for $\boldsymbol{d}$ is solved numerically by meshing the particle surface with isoparametric 6-node curved triangular boundary elements. The IBIM is applied here to the cases of a spherical and an ellipsoidal particle. The particle is either moving in a fluid at rest or settling in a gravity field, or entrained in a shear flow. A linear and a quadratic shear flow are considered. Various results for the friction factors and for the particle velocity are obtained. Relationships between friction coefficient (torque due to rotation and force due to translation), which are classical for a no-slip plane, do not apply here. The difference appears clearly in figure 6 for a sphere and in figure 11 for the ellipsoid. 

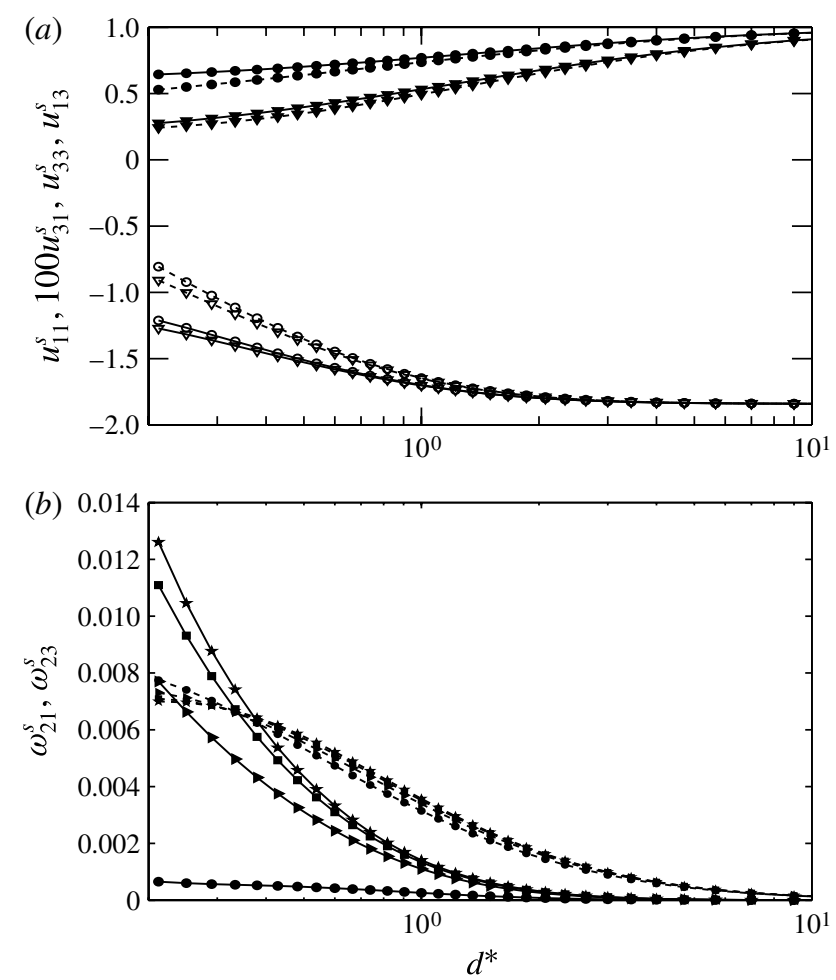

FiguRE 13. (a) Translational mobilities versus $d^{*}$ for $\lambda=1 / 30$ (dashed lines) and $\lambda=1 / 3$ (solid lines): $u_{11}^{s}(\bullet), 100 u_{31}^{s}(\circ), u_{33}^{s}(\boldsymbol{\nabla})$ and $u_{13}^{s}(\nabla)$. (b) Angular mobilities $\omega_{21}^{s}$ (solid lines) and $\omega_{23}^{s}$ (dashed lines) for: $\lambda=1 / 3(\bullet), \lambda=0.1(\boldsymbol{\Delta}), \lambda=1 / 30(\boldsymbol{\square})$ and $\lambda=0.01(\star)$.

The proposed IBIM also offers a way to obtain various formulae for an arbitrarily shaped particle entrained in a flow field near a porous slab. For example, asymptotic solutions of the integral equation for large particle-wall distance could provide asymptotic expansions for the force and torque. A study of this issue is currently in progress.

Obviously, the fluid velocity and pressure fields could also be subsequently calculated by (3.8) and (3.9), respectively, once $\boldsymbol{d}$ is known. The coupling of suspension motion with thermal or chemical advection or similar problems could then be considered.

An ultimate goal would be to use the IBIM in applications involving a number of particles, in view of applications in microfiltration, separation techniques in analytical chemistry and various situations that are relevant in biology.

Appendix A. How to obtain the stress $f=\sigma \cdot n$ from the auxiliary surface density of forces $d$

Assuming that $\boldsymbol{d}$ has been obtained by inverting (3.10), we show in this appendix how to obtain the surface stress $\boldsymbol{f}=\boldsymbol{\sigma} \cdot \boldsymbol{n}$. Using for the derived Green's tensor $\boldsymbol{G}$ and associated pressure vector $\boldsymbol{H}$ the decompositions (3.2) and (3.4), respectively,

$$
G(x, y)=G^{\infty}(x, y)+R(x, y), \quad H(x, y)=\frac{2(x-y)}{|x-y|^{3}}+Q(x, y),
$$



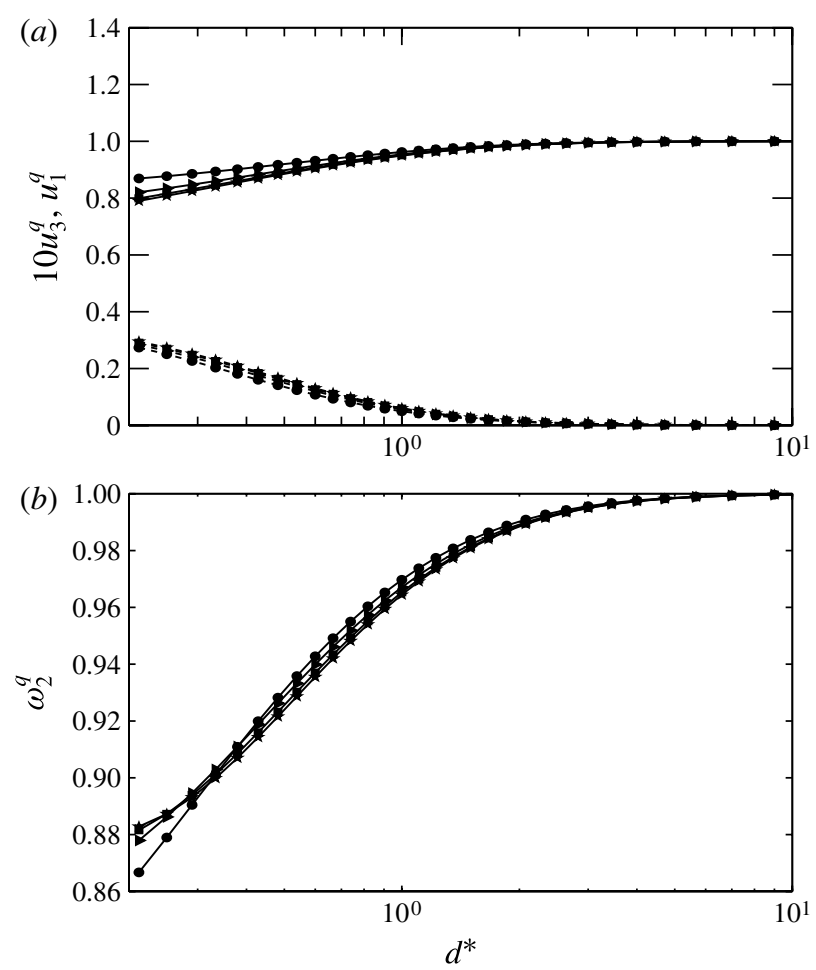

FIGURE 14. Case of quadratic ambient shear flow for $\lambda=1 / 3(\bullet), \lambda=0.1(\boldsymbol{\Delta}), \lambda=1 / 30($ and $\lambda=0.01(\star)$ : (a) $10 u_{3}^{q}$ (dashed lines) and $u_{1}^{q}$ (solid lines); $(b)$ angular mobility $\omega_{2}^{q}$.

the velocity field $\boldsymbol{u}$ and pressure field $p$ (given by (3.8) and (3.9)) are obtained as $\boldsymbol{u}=\boldsymbol{v}^{\infty}+\boldsymbol{v}$ and $p=q^{\infty}+q$, with the following integral representations in the fluid domain:

$$
\begin{gathered}
\boldsymbol{v}^{\infty}(\boldsymbol{x})=-\frac{1}{8 \pi \mu} \int_{S} \boldsymbol{G}^{\infty}(\boldsymbol{x}, \boldsymbol{y}) \cdot \boldsymbol{d}(\boldsymbol{y}) \mathrm{d} S, \quad q^{\infty}(\boldsymbol{x})=-\frac{1}{4 \pi} \int_{S} \frac{(\boldsymbol{x}-\boldsymbol{y})}{|\boldsymbol{x}-\boldsymbol{y}|^{3}} \cdot \boldsymbol{d}(\boldsymbol{y}) \mathrm{d} S, \\
\boldsymbol{v}(\boldsymbol{x})=-\frac{1}{8 \pi \mu} \int_{S} \boldsymbol{R}(\boldsymbol{x}, \boldsymbol{y}) \cdot \boldsymbol{d}(\boldsymbol{y}) \mathrm{d} S, \quad q(\boldsymbol{x})=-\frac{1}{8 \pi} \int_{S} \boldsymbol{Q}(\boldsymbol{x}, \boldsymbol{y}) \cdot \boldsymbol{d}(\boldsymbol{y}) \mathrm{d} S .
\end{gathered}
$$

The Stokes flow $\left(\boldsymbol{v}^{\infty}, q^{\infty}\right)$ is produced by distributing free-space stokeslet with density $\boldsymbol{d}$ on the particle $S$ where it exerts the stress $\boldsymbol{f}^{\infty}$. The Stokes flow $(\boldsymbol{v}, q)$ is highly regular in the limit when $\boldsymbol{x}$ tends to the surface $S$, so that the stress $\boldsymbol{f}-\boldsymbol{f}^{\infty}$ there is obtained from simple differentiation of $\boldsymbol{v}$ and use of (A 3) for the pressure. More precisely, elementary manipulations using (A 3) show that

$$
\left[\boldsymbol{f}-\boldsymbol{f}^{\infty}\right](\boldsymbol{x})=\frac{1}{8 \pi}\left\{\int_{S}\left[\delta_{i j} P_{k}(\boldsymbol{x}, \boldsymbol{y})-\frac{\left.\partial R_{j k}(\boldsymbol{x}, \boldsymbol{y})\right)}{\partial x_{i}}-\frac{\partial R_{i k}(\boldsymbol{x}, \boldsymbol{y})}{\partial x_{j}}\right] d_{k}(\boldsymbol{y}) \mathrm{d} S\right\} \boldsymbol{n}(\boldsymbol{x}) \cdot \boldsymbol{e}_{j} \boldsymbol{e}_{i},
$$

where the summation over repeated indices is used. The required stress $f^{\infty}$ produced by the Stokes flow $\left(\boldsymbol{v}^{\infty}, q^{\infty}\right)$ obeys on $S$ the following boundary integral equation (see 


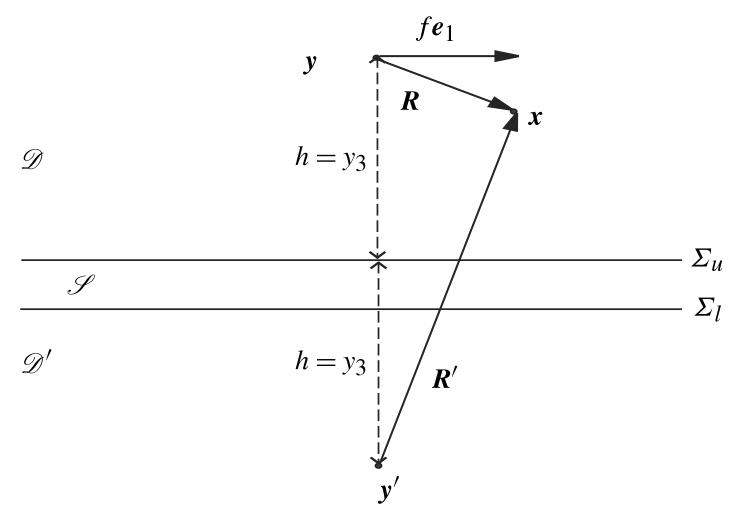

FIGURE 15. Stokeslet located at the pole $\boldsymbol{y}$ near a porous slab with $h=\boldsymbol{y} \cdot \boldsymbol{e}_{3}>0$. The symmetric point to (so-called image of) $\boldsymbol{y}$ with respect to the upper porous slab boundary $\Sigma_{u}$ is denoted by $\boldsymbol{y}^{\prime}$.

Pozrikidis 1992; Sellier 2010):

$$
\begin{aligned}
&- \frac{1}{8 \pi \mu} \int_{S} G^{\infty}(\boldsymbol{x}, \boldsymbol{y}) \cdot \boldsymbol{f}^{\infty}(\boldsymbol{y}) \mathrm{d} S \\
& \quad=\boldsymbol{v}^{\infty}(\boldsymbol{x})+\frac{3}{4 \pi} \int_{S} \frac{\left[\boldsymbol{v}^{\infty}(\boldsymbol{y})-\boldsymbol{v}^{\infty}(\boldsymbol{x})\right] \cdot(\boldsymbol{y}-\boldsymbol{x})(\boldsymbol{y}-\boldsymbol{x}) \cdot \boldsymbol{n}(\boldsymbol{y})(\boldsymbol{y}-\boldsymbol{x})}{|\boldsymbol{y}-\boldsymbol{x}|^{5}} \mathrm{~d} S \text { for } \boldsymbol{x} \text { on } S .
\end{aligned}
$$

Accordingly, one obtains $\boldsymbol{f}=\boldsymbol{f}-\boldsymbol{f}^{\infty}+\boldsymbol{f}^{\infty}$ by using (A 4) for $\boldsymbol{f}-\boldsymbol{f}^{\infty}$ and solving (A 5) for $f^{\infty}$, in conjunction with the integral representation (A 2) to compute $v^{\infty}$ also on $S$.

\section{Appendix B. Derivation of the Green's tensor}

In this appendix, we derive the expression of the Green's tensor $\left(\boldsymbol{V}^{(k)}(\boldsymbol{x}, \boldsymbol{y})\right.$, $\left.P^{(k)}(\boldsymbol{x}, \boldsymbol{y})\right)$, at a point $\boldsymbol{x}$ located in either $\mathscr{D}, \mathscr{S}$ or $\mathscr{D}^{\prime}$, due to a unit point force $f \boldsymbol{e}_{k}$ located at $\boldsymbol{y}$ in $\mathscr{D}$ (see figure 15).

\section{B.1. Governing equations}

The governing equations for $\boldsymbol{x}$ in the fluid domain $\mathscr{D}$ are the Stokes equations with an added point force singularity at $\boldsymbol{y}$ represented as a Dirac $\delta$ function with

$$
\boldsymbol{\nabla} \cdot \boldsymbol{V}^{(k)}(\boldsymbol{x}, \boldsymbol{y})=0, \quad \mu \nabla^{2} \boldsymbol{V}^{(k)}(\boldsymbol{x}, \boldsymbol{y})-\nabla P^{(k)}(\boldsymbol{x}, \boldsymbol{y})=-f \delta(\boldsymbol{x}-\boldsymbol{y}) \boldsymbol{e}_{k} .
$$

For $\boldsymbol{x}$ in the porous slab $\mathscr{S}$, Darcy's equations hold:

$$
\boldsymbol{V}^{(k)}=-\frac{K}{\mu} \nabla P^{(k)}, \quad \nabla \cdot \boldsymbol{V}^{(k)}=0 .
$$

Finally, for $\boldsymbol{x}$ in the fluid domain $\mathscr{D}^{\prime}$, one requires that

$$
\boldsymbol{\nabla} \cdot \boldsymbol{V}^{(k)}(\boldsymbol{x}, \boldsymbol{y})=0, \quad \mu \nabla^{2} \boldsymbol{V}^{(k)}(\boldsymbol{x}, \boldsymbol{y})-\nabla P^{(k)}(\boldsymbol{x}, \boldsymbol{y})=\mathbf{0} .
$$

The boundary conditions coupling these domains are

$$
\begin{array}{cl}
P^{(k)}\left(\Sigma_{u}^{+}\right)=P^{(k)}\left(\Sigma_{u}^{-}\right), & P^{(k)}\left(\Sigma_{l}^{+}\right)=P^{(k)}\left(\Sigma_{l}^{-}\right), \\
{\left[\boldsymbol{V}^{(k)} \cdot \boldsymbol{e}_{3}\right]\left(\Sigma_{u}^{+}\right)=\left[\boldsymbol{V}^{(k)} \cdot \boldsymbol{e}_{3}\right]\left(\Sigma_{u}^{-}\right),} & {\left[\boldsymbol{V}^{(k)} \cdot \boldsymbol{e}_{3}\right]\left(\Sigma_{l}^{+}\right)=\left[\boldsymbol{V}^{(k)} \cdot \boldsymbol{e}_{3}\right]\left(\Sigma_{l}^{-}\right),}
\end{array}
$$




$$
\begin{aligned}
& {\left[\frac{\partial \boldsymbol{V}^{(k)}}{\partial x_{3}} \cdot \boldsymbol{e}_{\beta}\right]\left(\Sigma_{u}^{+}\right)=\frac{\sigma}{\sqrt{K}}\left[\boldsymbol{V}^{(k)}\left(\Sigma_{u}^{+}\right)-\boldsymbol{V}^{(k)}\left(\Sigma_{u}^{-}\right)\right] \cdot \boldsymbol{e}_{\beta}, \quad(\beta=1,2),} \\
& {\left[\frac{\partial \boldsymbol{V}^{(k)}}{\partial x_{3}} \cdot \boldsymbol{e}_{\beta}\right]\left(\Sigma_{l}^{-}\right)=-\frac{\sigma}{\sqrt{K}}\left[\boldsymbol{V}^{(k)}\left(\Sigma_{l}^{-}\right)-\boldsymbol{V}^{(k)}\left(\Sigma_{l}^{+}\right)\right] \cdot \boldsymbol{e}_{\beta}, \quad(\beta=1,2),}
\end{aligned}
$$

where $\Sigma_{u}^{-}, \Sigma_{l}^{+}$, respectively, denote the sides of $\Sigma_{u}, \Sigma_{l}$ facing $\mathscr{S}$, and $\Sigma_{u}^{+}, \Sigma_{l}^{-}$those facing the fluid regions $\mathscr{D}, \mathscr{D}^{\prime}$. Finally, one adds the far-field behaviour

$$
\left(\boldsymbol{V}^{(k)}, P^{(k)}\right) \rightarrow(\mathbf{0}, 0) \quad \text { as }|\boldsymbol{x}| \rightarrow \infty .
$$

For a prescribed value of $k=1,2,3$, one has to solve (B 1)-(B 3) with boundary conditions (B 4)-(B 8).

\section{B.2. Analytical solutions in Fourier space}

As depicted in figure 15, we set $\boldsymbol{R}=\boldsymbol{x}-\boldsymbol{y}$ and $\boldsymbol{R}^{\prime}=\boldsymbol{x}-\boldsymbol{y}^{\prime}$, where $\boldsymbol{y}^{\prime}$ is the symmetric point of $\boldsymbol{y}$ with respect to $\Sigma_{u}$. Moreover, $R=|\boldsymbol{R}|, R^{\prime}=\left|\boldsymbol{R}^{\prime}\right|, R_{j}=\boldsymbol{R} \cdot \boldsymbol{e}_{j}$ and $R_{j}^{\prime}=\boldsymbol{R}^{\prime} \cdot \boldsymbol{e}_{j}$, so that $R_{3}=x_{3}-y_{3}$ and $R_{3}^{\prime}=x_{3}+y_{3}$. In solving the problem (B 1)-(B 8) we use the following two-dimensional Fourier transform:

$$
\hat{g}\left(\lambda_{1}, \lambda_{2} ; R_{3}^{\prime}\right)=\frac{1}{2 \pi} \int_{-\infty}^{\infty} \int_{-\infty}^{\infty} g\left(R_{1}^{\prime}, R_{2}^{\prime}, R_{3}^{\prime}\right) \mathrm{e}^{\mathrm{i}\left(\lambda_{1} R_{1}^{\prime}+\lambda_{2} R_{2}^{\prime}\right)} \mathrm{d} R_{1}^{\prime} \mathrm{d} R_{2}^{\prime} .
$$

Extending the approach of Blake (1971) and Elasmi \& Feuillebois (2001), we write the solution in the liquid domain $\mathscr{D}$ as the sum of a stokeslet with intensity $f \boldsymbol{e}_{k}$ located at $\boldsymbol{y}$, its image stokeslet with intensity $-f \boldsymbol{e}_{k}$ located at $\boldsymbol{y}^{\prime}$ (the previously defined symmetric point of $\boldsymbol{y}$ with respect to $\Sigma_{u}$ ) and a supplementary regular Stokes flow field with velocity $W_{j}^{(k)} \boldsymbol{e}_{j}$ and pressure $s^{(k)}$. In other words, one adopts in $\mathscr{D}$ the decompositions

$$
\begin{aligned}
V_{j}^{(k)} & =\boldsymbol{V}^{(k)} \cdot \boldsymbol{e}_{j}=\frac{f}{8 \pi \mu}\left\{\delta_{j k}\left(\frac{1}{R}-\frac{1}{R^{\prime}}\right)+\frac{R_{j} R_{k}}{R^{3}}-\frac{R_{j}^{\prime} R_{k}^{\prime}}{R^{\prime 3}}\right\}+W_{j}^{(k)}, \\
P^{(k)} & =\frac{f}{4 \pi}\left(\frac{R_{k}}{R^{3}}-\frac{R_{k}^{\prime}}{R^{\prime 3}}\right)+s^{(k)} .
\end{aligned}
$$

Taking the Fourier transform of the second equation in (B 1), using (B 8) and solving, the Fourier transforms of the supplementary flow pressure and velocity are found to be

$$
\begin{gathered}
\hat{s}^{(k)}=2 \mu B^{(k)} \mathrm{e}^{-\left(R_{3}^{\prime}-h\right) \xi}, \\
\widehat{W}_{j}^{(k)}=\left[B_{j}^{(k)}+B^{(k)}\left(\frac{\mathrm{i} \lambda_{\alpha}}{\xi} \delta_{\alpha j}+\delta_{3 j}\right)\left(R_{3}^{\prime}-h\right)\right] \mathrm{e}^{-\left(R_{3}^{\prime}-h\right) \xi},
\end{gathered}
$$

with the definitions $h=y_{3}, \xi=\sqrt{\lambda_{1}^{2}+\lambda_{2}^{2}}$ and $B_{j}^{(k)}=\widehat{W}_{j}^{(k)}\left(\Sigma_{u}^{+}\right)$for $j=1,2,3$. In addition, the first (continuity) equation (B 1) provides the relationship

$$
B^{(k)}=\mathrm{i} \lambda_{\alpha} B_{\alpha}^{(k)}+\xi B_{3}^{(k)}, \quad \alpha=1,2 .
$$

Here $k=1,2,3$ is given and one looks at four complex quantities $B^{(k)}$ and $B_{j}^{(k)}(j=1,2,3)$, which also depend on the solution in the porous medium $\mathscr{S}$ and liquid domain $\mathscr{D}^{\prime}$ through the coupled boundary conditions (B 4)-(B 7).

The pressure in the porous slab $\mathscr{S}$ being harmonic, its Fourier transform reads

$$
\widehat{P}^{(k)}=\widehat{P}^{(k)}\left(\Sigma_{u}^{-}\right) \frac{\sinh \left[\left(R_{3}^{\prime}-h+e\right) \xi\right]}{\sinh (\xi e)}-\widehat{P}^{(k)}\left(\Sigma_{l}^{+}\right) \frac{\sinh \left[\left(R_{3}^{\prime}-h\right) \xi\right]}{\sinh (\xi e)} .
$$


By virtue of (B 2), one immediately obtains in the porous slab

$$
\begin{gathered}
\widehat{V}_{\beta}^{(k)}=\mathrm{i} \lambda_{\beta} \frac{K}{\mu} \widehat{P}^{(k)} \quad \text { for } \beta=1,2, \\
\widehat{V}_{3}^{(k)}=-\xi \frac{K}{\mu}\left\{\widehat{P}^{(k)}\left(\Sigma_{u}^{-}\right) \frac{\cosh \left[\left(R_{3}^{\prime}-h+e\right) \xi\right]}{\sinh (\xi e)}-\widehat{P}^{(k)}\left(\Sigma_{l}^{+}\right) \frac{\cosh \left[\left(R_{3}^{\prime}-h\right) \xi\right]}{\sinh (\xi e)}\right\} .
\end{gathered}
$$

In the fluid domain $\mathscr{D}^{\prime}$, like in $\mathscr{D}$, we take the Fourier transform of the homogeneous Stokes equations (B 3), use (B 8) and solve the resulting equation, which depends on $R_{3}^{\prime}$, to obtain there

$$
\begin{gathered}
\widehat{P}^{(k)}=2 \mu A^{(k)} \mathrm{e}^{\left(R_{3}^{\prime}-h+e\right) \xi}, \\
\widehat{V}_{j}^{(k)}=\left[A_{j}^{(k)}-A^{(k)}\left(\frac{\mathrm{i} \lambda_{\alpha}}{\xi} \delta_{\alpha j}-\delta_{3 j}\right)\left(R_{3}^{\prime}-h+e\right)\right] \mathrm{e}^{\left(R_{3}^{\prime}-h+e\right) \xi},
\end{gathered}
$$

with, exploiting the first equation (B 3),

$$
\begin{gathered}
A_{j}^{(k)}=\widehat{V}_{j}^{(k)}\left(\Sigma_{0}^{-}\right) \quad(j=1,2,3), \\
A^{(k)}=\mathrm{i} \lambda_{\alpha} A_{\alpha}^{(k)}-\xi A_{3}^{(k)} \quad(\alpha=1,2) .
\end{gathered}
$$

In summary, we end up with eight unknown quantities $\widehat{P}^{(k)}\left(\Sigma_{u}^{-}\right), \widehat{P}^{(k)}\left(\Sigma_{l}^{+}\right), B_{j}^{(k)}$ and $A_{j}^{(k)}(j=1,2,3)$ to be found from the eight coupled boundary conditions (B 4)-(B 7).

The boundary conditions (B 4), (B 5) and (B 7) on the lower interface $\Sigma_{l}$ first yield the relationships

$$
\begin{aligned}
& 2 \mu A^{(k)}=\widehat{P}^{(k)}\left(\Sigma_{l}^{+}\right), \quad A_{3}^{(k)}=-\xi \frac{K}{\mu}\left[\frac{\widehat{P}^{(k)}\left(\Sigma_{u}^{-}\right)}{\sinh (\xi e)}-\frac{\widehat{P}^{(k)}\left(\Sigma_{l}^{+}\right)}{\tanh (\xi e)}\right], \\
& \left(\xi+\frac{\sigma}{\sqrt{K}}\right) A_{\beta}^{(k)}=\frac{\mathrm{i} \lambda_{\beta}}{\xi} A^{(k)}+\mathrm{i} \lambda_{\beta} \frac{\sigma \sqrt{K}}{\mu} \widehat{P}^{(k)}\left(\Sigma_{l}^{+}\right) \quad \text { for } \beta=1,2 .
\end{aligned}
$$

Using the decomposition (B 11) and (B 12), the continuity of pressure (B 4) across the interface $\Sigma_{u}$ gives

$$
\widehat{P}^{(k)}\left(\Sigma_{u}^{-}\right)=2\left(\delta_{3 k} \xi \psi_{0}+\mu B^{(k)}\right),
$$

in which, setting $R_{0}=\left\{R_{1}^{2}+R_{2}^{2}+h^{2}\right\}^{1 / 2}$, we define the function $\psi_{0}$ as

$$
\psi_{0}=-\frac{f}{4 \pi} \frac{\mathrm{e}^{-h \xi}}{\xi}=\hat{\varphi}_{0}, \quad \varphi_{0}=-\frac{f}{4 \pi R_{0}} .
$$

Recalling the decomposition (B 10), one also obtains

$$
\begin{aligned}
V_{j}^{(k)}\left(\Sigma_{u}^{+}\right) & =-\frac{f h}{4 \pi \mu}\left(\delta_{j \alpha} \delta_{3 k}+\delta_{j 3} \delta_{\alpha k}\right) \frac{R_{\alpha}}{R_{0}^{3}}+W_{j}^{(k)}\left(\Sigma_{u}^{+}\right), \quad j=1,2,3, \\
\frac{\partial V_{\beta}^{(k)}}{\partial R_{3}^{\prime}}\left(\Sigma_{u}^{+}\right) & =\frac{f h}{4 \pi \mu}\left(\frac{\delta_{\beta k}}{R_{0}^{3}}+\frac{3 R_{\beta} R_{\alpha} \delta_{\alpha k}}{R_{0}^{5}}\right)+\frac{\partial W_{j}^{(k)}}{\partial R_{3}^{\prime}}\left(\Sigma_{u}^{+}\right), \quad \beta=1,2,
\end{aligned}
$$

where there is a summation over indices $\alpha=1,2$ in (B 26) and (B 27). Hence, by virtue of (B 13), one arrives at

$$
\begin{gathered}
\widehat{V}_{j}^{(k)}\left(\Sigma_{u}^{+}\right)=\mathrm{i} h \lambda_{\beta}\left[\delta_{j \beta} \delta_{3 k}+\delta_{j 3} \delta_{\beta k}\right] \psi_{0} / \mu+B_{j}^{(k)}, \\
\frac{\partial \widehat{V}_{\beta}^{(k)}}{\partial R_{3}^{\prime}}\left(\Sigma_{u}^{+}\right)=\left[\lambda_{\alpha} \lambda_{\beta} \delta_{\alpha k} h-2 \xi \delta_{\beta k}\right] \frac{\psi_{0}}{\mu}+\frac{\mathrm{i} \lambda_{\beta}}{\xi} B^{(k)}-\xi B_{\beta}^{(k)}, \quad \beta=1,2 .
\end{gathered}
$$


Employing (B 28) and (B 17) the first boundary condition (B 5) then becomes

$$
B_{3}^{(k)}=-\frac{\mathrm{i} h \lambda_{\beta} \delta_{\beta k} \psi_{0}}{\mu}-\frac{\xi K}{\mu \sinh (\xi e)}\left[\cosh (e \xi) \widehat{P}^{(k)}\left(\Sigma_{u}^{-}\right)-\widehat{P}^{(k)}\left(\Sigma_{l}^{+}\right)\right] .
$$

Finally, by virtue of (B 16), (B 28) and (B 29) the last boundary conditions (B 6) yield

$$
\begin{aligned}
{\left[\xi+\frac{\sigma}{\sqrt{K}}\right] B_{\beta}^{(k)}=} & \mathrm{i} \lambda_{\beta}\left\{\frac{B^{(k)}}{\xi}+\sigma \frac{\sqrt{K}}{\mu} P^{(k)}\left(\Sigma_{u}^{-}\right)\right\} \\
& +\left[\lambda_{\alpha} \lambda_{\beta} h \delta_{\alpha k}-2 \xi \delta_{\beta k}-\mathrm{i} h \frac{\sigma}{\sqrt{K}} \lambda_{\beta} \delta_{3 k}\right] \frac{\psi_{0}}{\mu} \quad \text { for } \beta=1,2 .
\end{aligned}
$$

For given lengths $e$, permeability $K>0$ and dimensionless slip coefficient $\sigma>0$, we further introduce the quantities

$$
\begin{aligned}
& \eta=\frac{\sqrt{K}}{\sqrt{K} \xi+\sigma}, \quad \theta=1+2 \sigma \sqrt{K} \xi, \quad \tau=\frac{2 K \xi}{\tanh (e \xi)}, \quad \tau^{\prime}=\frac{2 K \xi}{\sinh (e \xi)}, \\
& \gamma=2 \sqrt{K} \xi-h / \sqrt{K}, \quad \Lambda=[1+\xi(\tau+\theta \eta)]^{-1}, \quad S=\left(\xi \Lambda \tau^{\prime}\right)^{2} .
\end{aligned}
$$

Exploiting (B 21)-(B 23) makes it possible to obtain a link between $\widehat{P}^{(k)}\left(\Sigma_{u}^{-}\right)$and $\widehat{P}^{(k)}\left(\Sigma_{l}^{+}\right)$. Curtailing the details, one obtains

$$
\widehat{P}^{(k)}\left(\Sigma_{l}^{+}\right)=\xi \Lambda \tau^{\prime} \widehat{P}^{(k)}\left(\Sigma_{u}^{-}\right) .
$$

Now, inserting (B 24) in (B 31) gives

$$
B_{\beta}^{(k)}=\frac{\mathrm{i} \lambda_{\beta}}{\xi} \eta \theta B^{(k)}+\eta\left[\mathrm{i} h \lambda_{\beta} \delta_{3 k} \sigma \gamma-2 \delta_{\beta k} \xi+\lambda_{\alpha} \lambda_{\beta} \delta_{\alpha k}\right] \frac{\psi_{0}}{\mu} \quad \text { for } \beta=1,2 .
$$

Invoking (B 24), (B 34) and (B 35) makes it possible to rewrite (B 30) as

$$
B_{3}^{(k)}=\left(\frac{S}{\xi \Lambda}-\tau\right) B^{(k)}-\left[\delta_{3 k} \xi\left(\tau-\frac{S}{\xi \Lambda}\right)+\mathrm{i} h \lambda_{\beta} \delta_{\beta k}\right] \frac{\psi_{0}}{\mu} .
$$

Appealing to (B 14) and taking account of (B 35) and (B 36) then provides the key result:

$$
B^{(k)}=-\xi\left[\frac{i \lambda_{\beta} \delta_{\beta k}[h+\eta(2-h \xi)] \Lambda+\delta_{3 k} \xi \Lambda(\tau+\sigma \gamma \eta)-\delta_{3 k} S}{1-S}\right] \frac{\psi_{0}}{\mu} .
$$

The above result (B 37) permits us to obtain, in terms of the known function $\psi_{0}$, the quantities $B_{j}^{(k)}$ for $j=1,2,3$ by employing (B 35) and (B 36), the coefficients $\widehat{P}^{(k)}\left(\Sigma_{l}^{+}\right)$ and $\widehat{P}^{(k)}\left(\Sigma_{u}^{-}\right)$by combining (B 34) with (B 30), and finally the quantities $A_{j}^{(k)}$ and $A_{j}^{(k)}$ from (B 22) and (B 23).

\section{B.3. Solution in the liquid domain above the porous slab}

Let us illustrate the way to proceed by determining the regular pressure $s^{(k)}$ occurring in the decomposition (B 11) and having (two-dimensional) Fourier transform $\hat{s}^{(k)}$ given by (B 12). Using (B 25) and (B 37) then provides

$$
\hat{s}^{(k)}=-2 \xi\left[\frac{\mathrm{i} \lambda_{\beta} \delta_{\beta k}[h+\eta(2-h \xi)] \Lambda+\delta_{3 k} \xi \Lambda(\tau+\sigma \gamma \eta)-\delta_{3 k} S}{1-S}\right] \psi,
$$


where the function $\psi$ is defined as

$$
\psi=-\frac{f}{4 \pi} \frac{\mathrm{e}^{-R_{3}^{\prime} \xi}}{\xi}=\hat{\psi}^{\prime}, \quad \psi^{\prime}=-\frac{f}{4 \pi R^{\prime}} .
$$

In taking the inverse Fourier transform, it is useful to note that the function $g\left(R_{1}^{\prime}, R_{2}^{\prime}, R_{3}^{\prime}\right)$ having a two-dimensional Fourier transform $\hat{g}$ (defined by (B 9)) given by (with a summation over indices $\beta=1,2$ in (B 40))

$$
\hat{g}\left(\lambda_{1}, \lambda_{2} ; R_{3}^{\prime}\right)=-\left(\delta_{j 3} \xi+\mathrm{i} \lambda_{\beta} \delta_{\beta j}\right) \frac{f(\xi)}{\xi} \mathrm{e}^{-R_{3}^{\prime} \xi}, \quad \xi=\sqrt{\lambda_{1}^{2}+\lambda_{2}^{2}},
$$

is, denoting by $\mathrm{J}_{0}$ the zeroth-order Bessel function and setting $\rho=\sqrt{R_{1}^{\prime 2}+R_{2}^{\prime 2}}$,

$$
g\left(R_{1}^{\prime}, R_{2}^{\prime}, R_{3}^{\prime}\right)=\left(\delta_{j 3} \frac{\partial}{\partial R_{3}^{\prime}}+\delta_{\beta j} \frac{\partial}{\partial R_{j}^{\prime}}\right)\langle f\rangle, \quad\langle f\rangle=\int_{0}^{\infty} f(\xi) \mathrm{J}_{0}(\rho \xi) \mathrm{e}^{-R_{3}^{\prime} \xi} \mathrm{d} \xi .
$$

In expressing the results obtained for the regular pressure $s^{(k)}$ and velocity $W_{j}^{(k)}$ introduced by (B 10) and (B 11), it is worth defining the symbol $\mathscr{J}_{k l}=\delta_{k 1} \delta_{1 l}+\delta_{k 2} \delta_{2 l}$ $\delta_{k 3} \delta_{3 l}$ and the following functions:

$$
\begin{gathered}
f_{1}=\frac{2 \Lambda}{1-S}[(\eta \xi-1) h-2 \eta], \quad f_{2}=-\frac{2 \Lambda}{1-S}[2 \eta(1+\sigma \sqrt{K} \xi)+\tau], \quad f_{3}=-\frac{2 S}{1-S}, \\
f_{4}=\frac{2 \Lambda \eta}{1-S}[(\xi h-2) \eta-h], \quad f_{5}=\eta f_{2}, \quad f_{6}=\eta f_{3}, \quad f_{7}=-\frac{2}{\sqrt{K} \xi+\sigma} .
\end{gathered}
$$

Then, one obtains

$$
s^{(k)}=-\frac{f}{4 \pi} \frac{\partial}{\partial R_{3}^{\prime}}\left[\mathscr{J}_{k l} \frac{\partial\left\langle f_{1}\right\rangle}{\partial R_{l}^{\prime}}+\delta_{3 k} \frac{\partial\left\langle f_{2}\right\rangle}{\partial R_{3}^{\prime}}+\delta_{3 k}\left\langle f_{3}\right\rangle\right],
$$

and in a similar fashion $W_{j}^{(k)}=w_{j k} /(8 \pi)$ with $w_{j k}$ given by (3.15).

\section{B.4. Solution in the porous medium $\mathscr{S}$}

The Fourier transform $\widehat{P}^{(k)}$ of the pressure in the porous medium is obtained by combining (B 15), (B 24), (B 34) and (B 37). It reads

$$
\begin{aligned}
\widehat{P}^{(k)}= & 2\left[-\xi\left(\mathrm{i} \lambda_{\alpha} \delta_{\alpha k}-\xi \delta_{3 k}\right)+\delta_{3 k} \frac{\xi}{h}\right][h+(2-\xi h) \eta] \frac{\sinh \left[\xi\left(R_{3}^{\prime}-h+e\right)\right]}{\sinh (\xi e)} \frac{\Lambda \psi_{0}}{1-S} \\
& -2 \xi \Lambda \tau^{\prime}\left[-\xi\left(\mathrm{i} \lambda_{\alpha} \delta_{\alpha k}-\xi \delta_{3 k}\right)+\delta_{3 k} \frac{\xi}{h}\right][h+(2-\xi h) \eta] \frac{\sinh \left[\xi\left(R_{3}^{\prime}-h\right)\right]}{\sinh (\xi e)} \frac{\Lambda \psi_{0}}{1-S} \\
& -4 \delta_{3 k}\left(\frac{\xi \eta}{h}\right) \frac{\sinh \left[\xi\left(R_{3}^{\prime}-h+e\right)\right]}{\sinh (\xi e)} \frac{\Lambda \psi_{0}}{1-S} \\
& +4 \xi \Lambda \tau^{\prime} \delta_{3 k}\left(\frac{\xi \eta}{h}\right) \frac{\sinh \left[\xi\left(R_{3}^{\prime}-h\right)\right]}{\sinh (\xi e)} \frac{\Lambda \psi_{0}}{1-S} .
\end{aligned}
$$

In expressing the resulting pressure $P^{(k)}$ in the porous medium, it is useful to introduce the so-called Hankel transform of order zero of a function $g$, denoted by $\mathscr{H}_{0}(g)$ and 
defined as

$$
\mathscr{H}_{0}(g)=\int_{0}^{\infty} \mathrm{J}_{0}(\rho \xi) \xi g(\xi) \mathrm{d} \xi .
$$

Then one arrives at

$$
\begin{aligned}
P^{(k)}= & \frac{1}{2 K}\left[\left(\mathscr{J}_{k l} \frac{\partial}{\partial R_{l}^{\prime}}+\frac{\delta_{3 k}}{h}\right)\left(\Upsilon_{3}-\Upsilon_{2}\right)+\left(\frac{\partial}{\partial R_{k}^{\prime}}+\frac{\delta_{3 k}}{h}\right)\left(\Upsilon_{1}-\Upsilon_{4}\right)\right] \\
& -\frac{2}{K} \delta_{3 k}\left(\Upsilon_{5}-\Upsilon_{6}\right),
\end{aligned}
$$

with the functions $\Upsilon_{i}\left(\rho, R_{3}^{\prime}\right)(i=1, \ldots, 6)$ defined as

$$
\begin{aligned}
& \Upsilon_{1}\left(\rho, R_{3}^{\prime}\right)=\mathscr{H}_{0}\left(\frac{(2-\xi h) \eta+h}{1+\xi(\tau+\eta \theta)} \frac{\tau^{\prime} \psi_{1}}{1-S}\right), \\
& \Upsilon_{2}\left(\rho, R_{3}^{\prime}\right)=\mathscr{H}_{0}\left(\frac{(2-\xi h) \eta+h}{1+\xi(\tau+\eta \theta)} \frac{\tau^{\prime} \psi_{2}}{1-S}\right), \\
& \Upsilon_{3}\left(\rho, R_{3}^{\prime}\right)=\mathscr{H}_{0}\left(\frac{(2-\xi h) \eta+h}{\xi} \frac{S \psi_{3}}{1-S}\right), \\
& \Upsilon_{4}\left(\rho, R_{3}^{\prime}\right)=\mathscr{H}_{0}\left(\frac{(2-\xi h) \eta+h}{\xi} \frac{S \psi_{4}}{1-S}\right) \\
& \Upsilon_{5}\left(\rho, R_{3}^{\prime}\right)=\mathscr{H}_{0}\left(\frac{\eta \sinh \left[\xi\left(R_{3}^{\prime}-h+e\right)\right]}{h[1+\xi(\tau+\eta \theta)]} \frac{\tau^{\prime} \psi_{0}}{1-S}\right), \\
& \Upsilon_{6}\left(\rho, R_{3}^{\prime}\right)=\mathscr{H}_{0}\left(\frac{\eta \sinh \left[\xi\left(R_{3}^{\prime}-h\right)\right]}{h \xi} \frac{S \psi_{0}}{1-S}\right)
\end{aligned}
$$

where $\psi_{0}$ has been introduced by (B 25) and

$$
\psi_{1}=\psi_{0} \mathrm{e}^{\xi\left(R_{3}^{\prime}-h+e\right)}, \quad \psi_{2}=\psi_{0} \mathrm{e}^{-\xi\left(R_{3}^{\prime}-h+e\right)}, \quad \psi_{3}=\psi_{0} \mathrm{e}^{-\xi\left(R_{3}^{\prime}-h\right)}, \quad \psi_{4}=\psi_{0} \mathrm{e}^{\xi\left(R_{3}^{\prime}-h\right)}
$$

Of course, the velocity $\boldsymbol{V}^{(k)}$ in the porous medium is then immediately deduced from (B 47) by appealing to Darcy's law, i.e. to the first equation in (B 2).

\section{B.5. Solution in the liquid domain $\mathscr{D}^{\prime}$}

From (B 22), (B 34) and (B 24) it is clear that

$$
A^{(k)}=\xi \Lambda \tau^{\prime}\left(\delta_{3 k} \xi \frac{\psi_{0}}{\mu}+B^{(k)}\right) .
$$

Combining (B 23) with (B 22) then also provides

$$
\begin{gathered}
A_{3}^{(k)}=-\tau^{\prime}(1-\xi \Lambda \tau)\left(\delta_{3 k} \xi \frac{\psi_{0}}{\mu}+B^{(k)}\right), \\
A_{\beta}^{(k)}=\mathrm{i} \lambda_{\beta} \Lambda \tau^{\prime} \eta \theta\left(\delta_{3 k} \xi \frac{\psi}{\mu}+B^{(k)}\right) \quad \text { for } \beta=1,2 .
\end{gathered}
$$


Upon introducing the additional functions

$$
\begin{aligned}
& F_{1}\left(\rho, R_{3}^{\prime}\right)=\mathscr{H}_{0}\left(\frac{(2-\xi h) \eta+h}{\xi \tau^{\prime}} \frac{S \psi_{1}}{1-S}\right), \quad F_{2}\left(\rho, R_{3}^{\prime}\right)=\mathscr{H}_{0}\left(\frac{\eta}{\xi \tau^{\prime}} \frac{S \psi_{1}}{1-S}\right), \\
& F_{3}\left(\rho, R_{3}^{\prime}\right)=\mathscr{H}_{0}\left(\frac{(2-\xi h) \eta+h}{\xi \tau^{\prime}} \frac{S \eta \psi_{1}}{1-S}\right), \quad F_{4}\left(\rho, R_{3}^{\prime}\right)=\mathscr{H}_{0}\left(\frac{\eta^{2}}{\xi \tau^{\prime}} \frac{S \psi_{1}}{1-S}\right),
\end{aligned}
$$

it is then established that the pressure and the velocity components $V_{j}^{(k)}=\boldsymbol{V}^{(k)} \cdot \boldsymbol{e}_{j}$ below the porous slab are

$$
\begin{gathered}
\mu V_{j}^{(k)}=\mathbb{W}_{j}^{3}\left[\frac{\partial F_{1}}{\partial R_{k}^{\prime}}+\delta_{3 k}\left(\left(\frac{1}{h}-\frac{\partial}{\partial R_{3}^{\prime}}\right)\left(F_{1}-2 F_{2}\right)+\frac{h \sigma}{\sqrt{K}} \frac{\partial F_{2}}{\partial R_{3}^{\prime}}\right)\right] \\
+(e-h) \frac{\partial}{\partial R_{j}^{\prime}}\left[\frac{\partial F_{1}}{\partial R_{k}^{\prime}}+\delta_{3 k}\left(\left(\frac{1}{h}-\frac{\partial}{\partial R_{3}^{\prime}}\right)\left(F_{1}-2 F_{2}\right)+\frac{h \sigma}{\sqrt{K}} \frac{\partial F_{2}}{\partial R_{3}^{\prime}}\right)\right] \\
-\left(1+2 \sigma \sqrt{K} \frac{\partial}{\partial R_{3}^{\prime}}\right) \frac{\partial}{\partial R_{j}^{\prime}}\left[\frac{\partial F_{3}}{\partial R_{k}^{\prime}}+\delta_{3 k}\left(\left(\frac{1}{h}-\frac{\partial}{\partial R_{3}^{\prime}}\right)\left(F_{3}-2 F_{4}\right)+\frac{h \sigma}{\sqrt{K}} \frac{\partial F_{4}}{\partial R_{3}^{\prime}}\right)\right] \\
P^{(k)}=2 \frac{\partial}{\partial R_{3}^{\prime}}\left[\frac{\partial F_{1}}{\partial R_{k}^{\prime}}+\delta_{3 k}\left(\left(\frac{1}{h}-\frac{\partial}{\partial R_{3}^{\prime}}\right)\left(F_{1}-2 F_{2}\right)+\frac{h \sigma}{\sqrt{K}} \frac{\partial F_{2}}{\partial R_{3}^{\prime}}\right)\right],
\end{gathered}
$$

where the following condensed notation is employed:

$$
\mathbb{W}_{j}^{3}=R_{3}^{\prime} \frac{\partial}{\partial R_{j}^{\prime}}-\delta_{j 3} .
$$

\section{Appendix C. Formulae for the numerical calculation of the Green's tensor and its derivatives}

The integral in the inverse transform (3.14) has to be calculated numerically. As for the derivatives of the inverse transform (3.14) with respect to the components of $\boldsymbol{R}^{\prime}$, we use the classical formulae:

$$
\frac{\mathrm{dJ}_{0}(t)}{\mathrm{d} t}=-\mathrm{J}_{1}(t), \quad \frac{\mathrm{dJ}_{1}(t)}{\mathrm{d} t}=\mathrm{J}_{0}(t)-\frac{\mathrm{J}_{1}(t)}{t},
$$

for $t>0$, where $\mathrm{J}_{1}$ is the Bessel function of order one. We then obtain for $\rho>0$ :

$$
\begin{aligned}
\frac{\partial\langle g\rangle}{\partial R_{3}^{\prime}}=-\langle\xi g\rangle, \quad \frac{\partial\langle g\rangle}{\partial R_{\alpha}^{\prime}}=-\frac{R_{\alpha}^{\prime}}{\rho} \int_{0}^{\infty} \xi \mathrm{J}_{1}(\rho \xi) g(\xi) \mathrm{e}^{-R_{3}^{\prime} \xi} \mathrm{d} \xi \quad \text { for } \alpha=1,2, \\
\frac{\partial^{2}\langle g\rangle}{\partial R_{\alpha}^{\prime} \partial R_{\beta}^{\prime}}=-\frac{\delta_{\alpha \beta}}{\rho} \int_{0}^{\infty} \xi \mathrm{J}_{1}(\rho \xi) g(\xi) \mathrm{e}^{-R_{3}^{\prime} \xi} \mathrm{d} \xi+\frac{R_{\alpha}^{\prime} R_{\beta}^{\prime}}{\rho^{2}} \int_{0}^{\infty} \xi^{2} \\
\times\left[2 \frac{\mathrm{J}_{1}(\rho \xi)}{\rho \xi}-\mathrm{J}_{0}(\rho \xi)\right] g(\xi) \mathrm{e}^{-R_{3}^{\prime} \xi} \mathrm{d} \xi \quad \text { for } \alpha=1,2, \beta=1,2 .
\end{aligned}
$$

As stated above, the relationship (C 2b) holds for $\rho>0$. However, for $\boldsymbol{x} \neq \boldsymbol{y}$ we have $R_{3}^{\prime}>0$ but may have $\rho=0$. Exploiting the asymptotics for $t \ll 1$, i.e.

$$
\mathrm{J}_{0}(t)=1-t^{2} / 4+O\left(t^{3}\right), \quad \mathrm{J}_{1}(t)=t / 2-t^{3} / 16+O\left(t^{5}\right),
$$


the limit values of the derivatives in (C2) then become

$$
\frac{\partial\langle g\rangle}{\partial R_{\alpha}^{\prime}}=0, \quad \frac{\partial^{2}\langle g\rangle}{\partial R_{\alpha}^{\prime} \partial R_{\beta}^{\prime}}=-\frac{\delta_{\alpha \beta}}{2} \int_{0}^{\infty} \xi^{2} g(\xi) \mathrm{e}^{-R_{3}^{\prime} \xi} \mathrm{d} \xi \quad \text { for } \rho=0 .
$$

In summary, it is remarked that any integral to be evaluated takes the form

$$
\mathscr{I}(\langle g\rangle)=\int_{0}^{\infty} \mathscr{F}(\rho \xi) g(\xi) \mathrm{e}^{-R_{3}^{\prime} \xi} \mathrm{d} \xi \quad \text { for } R_{3}^{\prime}=x_{3}+y_{3}>0 .
$$

By the change of variable $\xi=-\log t$, this integral is transformed to one on a finite interval:

$$
\mathscr{I}(\langle g\rangle)=\int_{0}^{1} \mathscr{F}(-\rho \log t) g(-\log t) t_{3}^{R_{3}^{\prime}-1} \mathrm{~d} t .
$$

This is more easily calculated numerically by iteratively splitting the domain [0, 1], using Gauss-Legendre quadrature in each sub-domain (note that Gauss-Laguerre is not appropriate to calculate directly the integral in (C 5) since $R_{3}^{\prime}$ may be small) and stopping the iterative scheme as soon as a sufficient, say of order $10^{-16}$, accuracy is reached. As verification, whenever possible, comparisons have been made up to order $O\left(10^{-16}\right)$ with results obtained using the Maple computer algebra software.

\section{REFERENCES}

Beavers, G. \& Joseph, D. 1967 Boundary conditions at a naturally permeable wall. J. Fluid Mech. 30, 197-207.

Beavers, G. S., Sparrow, E. M. \& Masha, B. A. 1974 Boundary condition at a porous surface which bounds a fluid flow. AIChE J. 20, 596.

Belfort, G., DAVIS, R. H. \& ZYDNEY, L. 1994 The behaviour of suspensions and macromolecular solutions in crossflow microfiltration. J. Membr. Sci. 96, 1-58.

Blake, J. R. 1971 A note on the image system for a stokeslet in a no-slip boundary. Proc. Camb. Phil. Soc. 70, 303-310.

Bonnet, M. 1995 Boundary Integral Equation Methods for Solids and Fluids. Wiley.

Brebbia, C. A., Telles, J. C. L. \& Wrobel, L. C. 1984 Boundary Element Techniques. Theory and Applications in Engineering. Springer.

BRENNER, H. 1961 The slow motion of a sphere through a viscous fluid towards a plane surface. Chem. Engng Sci. 16, 242-251.

Burganos, V. N., Michalopoulou, A. C., Dassios, G. \& Payatakes, A. C. 1992 Creeping flow around and through a permeable sphere moving with constant velocity towards a solid wall: a revision. Chem. Engng Commun. 117, 85-88.

ChANDESRIS, M. \& JAMET, D. 2007 Boundary conditions at a fluid-porous interface: an a priori estimation of the stress jump coefficients. Intl J. Heat Mass Transfer 50, 3422-3436.

Chaoui, M. \& Feuillebois, F. 2003 Creeping flow around a sphere in a shear flow close to a wall. Q. J. Mech. Appl. Maths 56 (3), 381-410.

Davis, A. M. J., Kezirian, M. T. \& Brenner, H. 1994 On the Stokes-Einstein model of surface diffusion along solid surfaces: slip boundary conditions. J. Colloid Interface Sci. 1065, $129-140$.

Debbech, A., Elasmi, L. \& Feuillebois, F. 2010 The method of fundamental solution for the creeping flow around a sphere close to a membrane. Z. Angew. Math. Mech. 90 (12), 920-928.

Elasmi, L. \& Feuillebois, F. 2001 Green function for a Stokes flow near a porous slab. Z. Angew. Math. Mech. 81 (11), 743-752.

Elasmi, L. \& Feuillebois, F. 2003 Integral equation method for creeping flow around a solid body near a porous slab. Q. J. Mech. Appl. Maths 56 (2), 163-185. 
Goren, S. L. 1979 The hydrodynamic force resisting the approach of a sphere to a plane permeable wall. J. Colloid Interface Sci. 69 (1), 78-85.

Gradshteyn, I. S. \& RYZhik, I. M. 1965 Table of Integrals, Series, and Products. Academic Press.

HAPpel, J. \& BREnNeR, H. 1991 Low Reynolds Number Hydrodynamics. Kluwer.

HSU, R. \& GANATOS, P. 1994 Gravitational and zero-drag motion of a spheroid adjacent to an inclined plane at low Reynolds number. J. Fluid Mech. 268, 267-292.

Khabthani, S. 2004 Calcul des solutions élémentaires pour le problème de filtration. Master's thesis, École Polytechnique de Tunisie.

KhAKpour, M. \& VAFAI, K. 2008 Critical assessment of arterial transport models. Intl J. Heat Mass Transfer 51, 807-822.

Loussaief, H. 2008 Ecoulement de suspensions avec condition de glissement sur la paroi. Thèse, Université Pierre et Marie Curie, Paris 6.

Luo, H. \& PozRikidis, C. 2008 Effect of surface slip on Stokes flow past a spherical particle in infinite fluid and near a plane wall. J. Engng Maths 62 (1), 1-21.

Maude, A. D. 1961 End effects in a falling-sphere viscometer. Brit. J. Appl. Phys. 12, 293-295.

Michalopoulou, A. C., Burganos, V. N. \& Payatakes, A. C. 1992 Creeping axisymmetric flow around a solid particle near a permeable obstacle. AIChE J. 38 (8), 1213-1228.

NIR, A. 1981 On the departure of a sphere from contact with a permeable membrane. J. Engng Maths 15 (1), 65-75.

OchoA-TApia, J. A. \& WhitAker, S. $1995 a$ Momentum transfer at the boundary between a porous medium and a homogeneous fluid - I. Theoretical development. Intl J. Heat Mass Transfer 38 (14), 2635-2646.

OchoA-TApia, J. A. \& WhitaKer, S. $1995 b$ Momentum transfer at the boundary between a porous medium and a homogeneous fluid - II. Comparison with experiment. Intl J. Heat Mass Transfer 38 (14), 2647-2655.

Pasol, L., Sellier, A. \& Feuillebois, F. 2006 A sphere in a second degree polynomial creeping flow parallel to a wall. Q. J. Mech. Appl. Maths 59 (4), 587-614.

Payatakes, A. C. \& Dassios, G. 1987 Creeping flow around and through a permeable sphere moving with constant velocity towards a solid wall. Chem. Engng Commun. 58, 119-138.

POZRIKIDIS, C. 1992 Boundary Integral and Singularity Methods for Linearized Viscous Flow. Cambridge University Press.

ReZayat, M., Shippy, D. J. \& Rizzo, F. J. 1986 On time-harmonic elastic-wave analysis by the boundary element method for moderate to high frequencies. Comput. Meth. Appl. Mech. Engng 55, 349-367.

RICHARDSON, S. 1971 A model for the boundary condition of a porous material. Part 2. J. Fluid Mech. 49 (2), 327-336.

SAfFMAN, P. G. 1971 On the boundary condition at the surface of a porous medium. Stud. Appl. Maths 50, 93-101.

SELlier, A. 2005 Settling motion of interacting solid particles in the vicinity of a plane solid boundary. C. R. Méc. 33, 413-418.

SELLIER, A. 2010 Boundary element technique for slow viscous flows about particles. In Boundary Element Method in Engineering and Sciences (ed. M. H. Aliabadin \& P. H. Wen), Computational and Experimental Methods in Structures, vol. 4. World Scientific.

Sherwood, J. D. 1988 The force on a sphere pulled away from a permeable half-space. Physico-Chem. Hydrodyn. 10 (1), 3-12.

TAYLOR, G. I. 1971 A model for the boundary condition of a porous material. Part 1. J. Fluid Mech. 49 (2), 319-326.

Valdés-Parada, F. J., Goyeau, B. \& Ochoa-tapia, J. A. 2007 Jump momentum boundary condition at a fluid-porous dividing surface: derivation of the closure problem. Chem. Engng Sci. 62, 4025-4039.

Wahlund, K. G. \& Giddings, J. C. 1987 Properties of an asymmetrical flow field-flow fractionation channel having one permeable wall. Analyt. Chem. 59, 1332-1339. 